\title{
ON THE TRACE FORMULA FOR HECKE OPERATORS ON CONGRUENCE SUBGROUPS, II
}

\author{
ALEXANDRU A. POPA
}

\begin{abstract}
In a previous paper, we obtained a general trace formula for double coset operators acting on modular forms for congruence subgroups, expressed as a sum over conjugacy classes. Here we specialize it to the congruence subgroups $\Gamma_{0}(N)$ and $\Gamma_{1}(N)$, obtaining explicit formulas in terms of class numbers for the trace of a composition of Hecke and Atkin-Lehner operators. The formulas are among the simplest in the literature, and hold without any restriction on the index of the operators. We give two applications of the trace formula for $\Gamma_{1}(N)$ : we determine explicit trace forms for $\Gamma_{0}(4)$ with Nebentypus, and we compute the limit of the trace of a fixed Hecke operator as the level $N$ tends to infinity.
\end{abstract}

\section{INTRODUCTION}

In [7], we gave a short proof of a trace formula for Hecke operators on modular forms of weight $k \geqslant 2$ for finite index subgroups of $\mathrm{SL}_{2}(\mathbb{Z})$. The formula expresses the following combination of traces

$$
\operatorname{Tr}\left([\Sigma], M_{k}(\Gamma, \chi)+S_{k}(\Gamma, \chi)\right):=\operatorname{Tr}\left([\Sigma], M_{k}(\Gamma, \chi)\right)+\operatorname{Tr}\left([\Sigma], S_{k}(\Gamma, \chi)\right),
$$

as a sum over conjugacy classes of simple factors (the notation and the precise formula is reviewed in Section 2). In this paper we specialize $\Gamma$ to be one of the congruence subgroups $\Gamma_{0}(N)$ or $\Gamma_{1}(N)$, and we obtain explicit trace formulas in terms of class numbers. These formulas are among the simplest in the extensive literature, requiring one extra ingredient compared to the $\mathrm{SL}_{2}(\mathbb{Z})$ case: an arithmetic function which is multiplicative in the level $N$. For example, for $\Gamma=\Gamma_{0}(N)$ and for $\chi$ any character modulo $N$, we obtain for all $k \geqslant 2, N \geqslant 1$ and $n \geqslant 1$ :

$$
\begin{array}{r}
\operatorname{Tr}\left(T_{n}, M_{k}(\Gamma, \chi)+S_{k}(\Gamma, \chi)\right)=-\sum_{t \in \mathbb{Z}} p_{k-2}(t, n) \cdot \sum_{\substack{u\left|N \\
u^{2}\right| t^{2}-4 n}} H\left(\frac{4 n-t^{2}}{u^{2}}\right) C_{N, \chi}(u, t, n)+ \\
+\delta_{k, 2} \delta_{\chi, 1} \sum_{d \mid n,(d, N)=1} n / d
\end{array}
$$

where $p_{k-2}(t, n)$ is the Gegenbauer polynomial, $H(D)$ is the Kronecker-Hurwitz class number, extended to negative squares by $H\left(-u^{2}\right)=u / 2$ for $u>0$ and to negative non-squares by 0 , and $C_{N, \chi}(u, t, n)$ is a multiplicative function in the level $N$, defined in (2.18).

It was Zagier's insight that the trace formula for $\mathrm{SL}_{2}(\mathbb{Z})$ takes the simplest form when written for the linear combination (1.1), and he expressed it in terms of the above extension of the

2010 Mathematics Subject Classification. 11F11, 11F25.

Key words and phrases. Trace formula; Hecke operators; holomorphic modular forms. 
Kronecker-Hurwitz class numbers to all integers [17, 18]. Here we show, based on the formula proved in [7], that the same phenomenon happens for any congruence subgroup satisfying a mild assumption (Assumption 2.1), which is verified by the usual congruence subgroups.

While the combination of traces (1.1) yields the simplest formula, for applications, and to compare it with the existing literature, it is useful to have a formula on the cuspidal subspace alone. The second goal of this paper is to extract the cuspidal trace from (1.1), by computing the trace on the Eisenstein subspace. We perform this computation for an arbitrary Fuchsian group of the first kind with cups, since the formula may be of independent interest, and since we expect the resulting formula for the cuspidal trace to hold for such a Fuchsian group as well.

The trace formula for Hecke operators on spaces of modular forms for Fuchsian groups has a long history, starting with the celebrated papers of Eichler [3] and Selberg [12]. The existing approaches lead to trace formulas on the cuspidal subspace alone, and the different types of conjugacy classes need separate treatments $[3,4,15,11,6,17]$, whereas in our approach all conjugacy classes are treated uniformly through a simple invariant. Existing formulas are quite complicated, and the index of the Hecke operators is usually assumed coprime with the level. Among the simplest and most general is the trace formula for $\Gamma_{0}(N)$ with Nebentypus proved by Oesterlé by analytic means $[2,6]$, an equivalent form of which we recover in Theorem 3.

We also obtain new formulas for the trace of a composition of Hecke and Atkin-Lehner operators for $\Gamma_{0}(N)$, both in terms of the regular class numbers, and in terms of the Kronecker-Hurwitz class numbers, and we impose no restrictions on the index of the operators involved. A previous such formula was given by Skoruppa and Zagier [16], assuming the index of the Hecke operator coprime with the level. We also include a trace formula for $\Gamma_{1}(N)$, which turns out to be simpler than for $\Gamma_{0}(N)$, and which is the first such formula available in the literature.

We include two applications that illustrate the versatility of our trace formulas. Specializing the trace formula for $\Gamma_{1}(N)$ to $N=4$ we obtain that

$$
\sum_{n \text { even }}\left(\sum_{\substack{t^{2} \leqslant 4 n \\ 4 \mid t-n-1}} p_{k-2}(t, n) H\left(4 n-t^{2}\right)+\sum_{\substack{n=a d \\ a \text { odd }}} \min (a, d)^{k-1}-\delta_{k, 2} \sum_{\substack{n=a d \\ a \text { odd }}} d\right) q^{n}
$$

is a cusp form in $S_{k}\left(\Gamma_{0}(4)\right)$ if $k \geqslant 2$ is even. The coefficients of this modular form are the traces $-\operatorname{Tr}\left(T_{n}, S_{k}\left(\Gamma_{0}(4)\right)\right.$ for $n$ even, and we obtain also an explicit generating series for the traces of $T_{n}$ with $n$ odd (see Corollary 2 in Section 2.4). While the explicit cusp form above seems not to have appeared before, the explicit generating series we get for odd coefficients was conjectured to be a cusp form by H. Cohen more than 30 years ago [1]. Although Cohen suggested that this modular form was related to the "trace form", whose coefficients are the traces of Hecke operators, the conjecture was only recently proved by different methods by M. H. Mertens [5]. In Corollary 2 we also compute explicitly the trace form for $S_{k}\left(\Gamma_{0}(4), \chi\right)$ if $k$ is odd and $\chi$ is the nontrivial character modulo 4. For $k=2$ or $k=3$, the corresponding spaces of cusp forms are trivial, and these formulas reduce to class number relations similar to the Kronecker-Hurwitz formula.

As another application, we take the limit in the trace formula for $\Gamma_{1}(N)$ as $N \rightarrow \infty$. We find that the trace of a fixed Hecke operator $T_{n}$ stabilizes, and the result is suprisingly independent of 
$n>1$ and $k \geqslant 2$ :

$$
\lim _{\substack{N \rightarrow \infty \\(N, n-1)=1}} \frac{\operatorname{Tr}\left(T_{n}, S_{k}\left(\Gamma_{1}(N)\right)\right)}{\varphi(N)}=-\frac{1}{2}
$$

(in fact for $N>2 n+2$ coprime to $n-1$ and $k>2$ we have equality). Such limit forms of the trace formula were used by Serre to prove equidistribution results for Hecke eigenvalues of a fixed Hecke operator on $\Gamma_{0}(N)$, as the level and weight tend to infinity [13]. Such limits also appear naturally in work of F. Rădulescu [10], in the context of certain distributions on $\mathrm{SL}_{2}(\mathbb{Z})$.

The paper is organized as follows. In Section 2 we state the main results: after recalling the main result of [7] in Theorem 1, the general trace formula on the cuspidal space is stated in Theorem 2. In $\S 2.2$ we show how to derive from it explicit versions in terms of class numbers, under an additional assumption. The group $\Gamma_{0}(N)$ satisfies this assumption, yielding the explicit formulas in Theorems 3 and 4. The trace formula for $\Gamma_{1}(N)$ is given in Theorem 5 , and its applications in Section 2.4.

In Section 3 we prove a trace formula on the Eisenstein subspace for a general Fuchsian group of the first kind (Theorem 3.5), and use it to prove Theorem 2. Finally, in Section 4 we compute the arithmetic functions entering Theorem 2 in the case of $\Gamma_{0}(N)$, thus proving Theorems 3 and 4 . Acknowledgements. This work was partly supported by the the European Community grant PIRG05-GA-2009-248569 and by the CNCS grant TE-2014-4-2077. Part of this work was completed during several visits at MPIM in Bonn, whose support I gratefully acknowledge.

\section{Statement of Results}

Let $\Gamma$ be a finite index subgroup of $\Gamma_{1}=\mathrm{SL}_{2}(\mathbb{Z})$ and let $\Sigma$ be a double coset contained in the commensurator $\widetilde{\Gamma} \subset \mathrm{GL}_{2}^{+}(\mathbb{R})$. If $\chi$ is a character of $\Gamma$ with kernel of finite index, the action of the double coset operator $[\Sigma]$ on $M_{k}(\Gamma, \chi)$ is defined using a multiplicative function $\tilde{\chi}$ on the semigroup generated by $\Gamma$ and $\Sigma$ inside $\widetilde{\Gamma}$, such that $\left.\widetilde{\chi}\right|_{\Gamma}=\chi^{-1}$, namely

$$
\tilde{\chi}\left(\gamma \sigma \gamma^{\prime}\right)=\chi^{-1}\left(\gamma \gamma^{\prime}\right) \widetilde{\chi}(\sigma), \quad \text { for all } \gamma \in \Gamma, \sigma \in \Sigma .
$$

A modular form $f \in M_{k}(\Gamma, \chi)$ satisfies $\left.f\right|_{k} \gamma=\chi(\gamma) f$, and the double coset operator $[\Sigma]$ acts by

$$
f\left|[\Sigma]=\sum_{\sigma \in \Gamma \backslash \Sigma} \operatorname{det} \sigma^{k-1} \cdot \tilde{\chi}(\sigma) \cdot f\right|_{k} \sigma
$$

where $\left.f\right|_{k} \gamma(z)=f(\gamma z)\left(c_{\gamma} z+d_{\gamma}\right)^{-k}$, and we write $\gamma=\left(\begin{array}{ll}a_{\gamma} & b_{\gamma} \\ c_{\gamma} & d_{\gamma}\end{array}\right)$ throughout the paper.

Example. Let $\Gamma$ be the congruence subgroup $\Gamma_{0}(N):=\left\{\gamma \in \Gamma_{1}: N \mid c_{\gamma}\right\}$, and let $\chi$ be a character modulo $N$ viewed as a character of $\Gamma_{0}(N)$ by $\chi(\gamma)=\chi\left(d_{\gamma}\right)$. The usual Hecke operators $T_{n}$ on $M_{k}(\Gamma, \chi)$ are associated to the double coset

$$
\Delta_{n}:=\left\{\sigma \in M_{2}(\mathbb{Z}): \operatorname{det} \sigma=n, N \mid c_{\sigma},\left(a_{\sigma}, N\right)=1\right\},
$$

and $\tilde{\chi}(\sigma)=\chi\left(a_{\sigma}\right)$ for $\sigma \in \Delta_{n}$. 
2.1. A general trace formula on the cuspidal subspace. For any subset $\mathcal{S}$ of $\mathrm{GL}_{2}^{+}(\mathbb{R})$, we denote by $\overline{\mathcal{S}}=(\mathcal{S} \cup-\mathcal{S}) /\{ \pm 1\} \subset \mathrm{GL}_{2}^{+}(\mathbb{R}) /\{ \pm 1\}$. By scaling $\Sigma$ we may assume that it is contained in the set $\mathcal{M}$ of integral matrices of positive determinant. For a $\bar{\Gamma}_{1}$ conjugacy class $X \subset \overline{\mathcal{M}}$, we choose any representative $M_{X} \in \mathcal{M}$ and we denote by $\Delta(X)=\operatorname{Tr}\left(M_{X}\right)^{2}-4 \operatorname{det}\left(M_{X}\right)$ the discriminant of the quadratic form associated to $M_{X}$, and by $\left|\operatorname{Stab}_{\bar{\Gamma}_{1}} M_{X}\right|$ the (possibly infinite) cardinality of the stabilizer of $M_{X}$ under conjugation by $\bar{\Gamma}_{1}$. We define the conjugacy class invariant

$$
\varepsilon(X)= \begin{cases}\frac{1}{6} & \text { if } M_{X} \text { scalar } \\ \frac{\operatorname{sgn} \Delta(X)}{\left|\operatorname{Stab}_{\bar{\Gamma}_{1}} M_{X}\right|} & \text { otherwise. }\end{cases}
$$

Explicitly $\varepsilon(X)$ is equal to: $1 / 6$ if $M_{X}$ is scalar; $-1 /\left|\operatorname{Stab}_{\bar{\Gamma}_{1}} M_{X}\right|$ if $M_{X}$ is elliptic; 1 if $M_{X}$ is hyperbolic fixing two cusps of $\Gamma_{1}$; and 0 otherwise.

To state the main result of [7], let $p_{w}(t, n)$ be the Gegenbauer polynomial, defined by the power series expansion $\left(1-t x+n x^{2}\right)^{-1}=\sum_{w \geqslant 0} p_{w}(t, n) x^{w}$.

Theorem $1([7])$. Let $\Gamma$ be a finite index subgroup of $\Gamma_{1}, k \geqslant 2$ an integer, $\chi$ a character of $\Gamma$ with kernel of finite index in $\Gamma$, and $\Sigma$ a double coset of $\Gamma$ such that $|\Gamma \backslash \Sigma|=\left|\Gamma_{1} \backslash \Gamma_{1} \Sigma\right|$. Assuming $\chi(-1)=(-1)^{k}$ if $-1 \in \Gamma$, we have

$$
\begin{array}{r}
\operatorname{Tr}\left([\Sigma], M_{k}(\Gamma, \chi)+S_{k}^{c}(\Gamma, \chi)\right)=\sum_{X} p_{k-2}\left(\operatorname{Tr} M_{X}, \operatorname{det} M_{X}\right) \mathcal{C}_{\Gamma, \Sigma}^{\chi}\left(M_{X}\right) \varepsilon(X) \\
+\delta_{k, 2} \delta_{\chi, 1} \sum_{\sigma \in \Gamma \backslash \Sigma} \widetilde{\chi}(\sigma),
\end{array}
$$

where the sum is over $\bar{\Gamma}_{1}$-conjugacy classes $X \subset \overline{\Gamma_{1} \Sigma \Gamma_{1}}$ with representative $M_{X} \in \Gamma_{1} \Sigma \Gamma_{1}$, and $d^{1}$

$$
\mathcal{C}_{\Gamma, \Sigma}^{\chi}(M):=\sum_{\substack{A \in \bar{\Gamma} \backslash \bar{\Gamma}_{1} \\ \pm A M A^{-1} \in \Sigma}}( \pm 1)^{k} \widetilde{\chi}\left( \pm A M A^{-1}\right) .
$$

The symbol $\delta_{a, b}$ is 1 if $a=b$ and 0 otherwise.

Here $\left.S_{k}^{c}(\Gamma, \chi)\right)$ is the space of anti-holomorphic cusp forms. From now on we assume for simplicity that $\Gamma, \Sigma$ and $\chi$ are invariant under conjugation by an order 2 element of determinant -1 (an assumption satisfied for most congruence subgroups, in particula for $\Gamma_{0}(N)$ ), so that we can replace the space $S_{k}^{c}(\Gamma, \chi)$ by $S_{k}(\Gamma, \chi)$ in the theorem (see [7, Remark 3.2]).

To state the formula on the cuspidal subspace, let $\Gamma_{\mathfrak{a}} \subset \Gamma$ and $\Sigma_{\mathfrak{a}} \subset \Sigma$ be the stabilizers of a cusp $\mathfrak{a}$ of $\Gamma$. Let $C(\Gamma)$ be a set of representatives for the $\Gamma$-equivalence classes of cusps and define the subset

$$
C(\Gamma, \chi)=\left\{\mathfrak{a} \in C(\Gamma): \chi(\gamma)=\operatorname{sgn}(\gamma)^{k} \text { if } \gamma \in \Gamma_{\mathfrak{a}}\right\},
$$

where $\operatorname{sgn}(\sigma):=\operatorname{sgn}(\operatorname{Tr} \sigma)$ denotes the sign of the eigenvalues of a parabolic or hyperbolic matrix $\sigma \in \mathrm{GL}_{2}^{+}(\mathbb{R})$.

\footnotetext{
${ }^{1}$ The same sign is chosen in all three places in (2.6). If $-1 \notin \Gamma$ at most one choice of signs is possible for each $A$, while if $-1 \in \Gamma$ both choices yield the same value for the summand.
} 
Theorem 2. With the notations of Theorem 1 we have

$$
\begin{array}{r}
\operatorname{Tr}\left([\Sigma], S_{k}(\Gamma, \chi)\right)=\frac{1}{2} \sum_{X, \Delta(X) \leqslant 0} p_{k-2}\left(\operatorname{Tr} M_{X}, \operatorname{det} M_{X}\right) \mathcal{C}_{\Gamma, \Sigma}^{\chi}\left(M_{X}\right) \varepsilon(X) \\
-\frac{1}{2} \sum_{\mathfrak{a} \in C(\Gamma, \chi)} \sum_{\sigma \in \Gamma_{\mathfrak{a}} \backslash \Sigma_{\mathfrak{a}} / \Gamma_{\mathfrak{a}}} \frac{\min \left(\left|\lambda_{\sigma}\right|,\left|\lambda_{\sigma}^{\prime}\right|\right)^{k-1}}{\left(\left|\lambda_{\sigma}\right|,\left|\lambda_{\sigma}^{\prime}\right|\right)} \operatorname{sgn}(\sigma)^{k} \widetilde{\chi}(\sigma)+\delta_{k, 2} \delta_{\chi, \mathbf{1}} \sum_{\sigma \in \Gamma \backslash \Sigma} \tilde{\chi}(\sigma),
\end{array}
$$

where $\lambda_{\sigma}, \lambda_{\sigma}^{\prime}$ are the eigenvalues of $\sigma$, and for $a, d \in \mathbb{R}$ with $a / d \in \mathbb{Q}$ we let $(a, d)$ be the unique positive number such that $\mathbb{Z} a+\mathbb{Z} d=\mathbb{Z}(a, d) .^{2}$

Remark. The sum over cusps in (2.7) can be written more explicitly as follows. Fix a scaling matrix $C_{\mathfrak{a}}$ for the cusp $\mathfrak{a}$, namely $C_{\mathfrak{a}} \mathfrak{a}=\infty$ and

$$
\{ \pm 1\} \cdot C_{\mathfrak{a}} \Gamma_{\mathfrak{a}} C_{\mathfrak{a}}^{-1}=\left\{ \pm\left(\begin{array}{cc}
1 & n \\
0 & 1
\end{array}\right): n \in \mathbb{Z}\right\} .
$$

For $a, d>0$ we set $\Sigma_{\mathfrak{a}}(a, d):=\left\{\sigma \in \Sigma_{\mathfrak{a}}: C_{\mathfrak{a}} \sigma C_{\mathfrak{a}}^{-1}=\operatorname{sgn}(\sigma)\left(\begin{array}{ll}a & * \\ 0 & d\end{array}\right)\right\}$ and

$$
\Phi_{\Gamma, \Sigma}^{\chi}(a, d):=\frac{1}{(a, d)} \sum_{\mathfrak{a} \in C(\Gamma, \chi)} \sum_{\sigma \in \Gamma_{\mathfrak{a}} \backslash \Sigma_{\mathfrak{a}}(a, d) / \Gamma_{\mathfrak{a}}} \operatorname{sgn}(\sigma)^{k} \widetilde{\chi}(\sigma) .
$$

We show in Theorem 3.5 that $\Phi_{\Gamma, \Sigma}^{\chi}(a, d)$ is symmetric in $a, d$, and we can rewrite the sum over cusps $\mathfrak{a}$ in $(2.7)$ as follows

$$
\sum_{a, d>0} \min (a, d)^{k-1} \Phi_{\Gamma, \Sigma}^{\chi}(a, d)
$$

It is the function $\Phi_{\Gamma, \Sigma}^{\chi}(a, d)$ that we will compute for $\Gamma=\Gamma_{0}(N)$.

2.2. Explicit trace formulas in terms of class numbers. The class numbers appear naturally in terms of the conjugacy class invariants $\varepsilon(X)$, and we first extend both the usual and the Kronecker-Hurwitz class numbers to all integers.

Let $\mathcal{M}_{n} \subset M_{2}(\mathbb{Z})$ denote the set of matrices of determinant $n$. The $\Gamma_{1}$-equivariant bijection $M=\left(\begin{array}{ll}a & b \\ c & d\end{array}\right) \leftrightarrow Q_{M}(x, y)=c x^{2}+(d-a) x y-b y^{2}$ between matrices of determinant $n$ and trace $t$ and binary quadratic forms of discriminant $t^{2}-4 n$ implies that for all $D$ and for $u \geqslant 1$ :

$$
\sum_{\substack{X \subset \overline{\mathcal{M}}_{n} \\
\Delta(X)=D, u \mid G_{X}}} \varepsilon(X)=\left\{\begin{array}{ll}
-2 H\left(-D / u^{2}\right) & \text { if } \operatorname{Tr}(X) \neq 0 \\
-H\left(-D / u^{2}\right) & \text { if } \operatorname{Tr} X=0
\end{array},\right.
$$

where $G_{X}$ is the content of the quadratic form $Q_{M}$ associated to any representative $M$ of $X$, and $H(D)$ is the Kronecker-Hurwitz class number for $D \geqslant 0$, as extended by Zagier to all $D[17,18]$. We recall that if $D>0, H(D)$ is the the number of $\Gamma_{1}$-equivalence classes of positive definite binary quadratic forms of discriminant $-D$, the forms with a stabilizer of order 2 or 3 in $\Gamma_{1}$ being counted with multiplicity $1 / 2$ or $1 / 3 ; H(0)=-1 / 12$; and if $D<0, H(D)$ is $-u / 2$ if $D=-u^{2}$ with $u \in \mathbb{Z}_{>0}$, and it is 0 if $-D$ is not a perfect square.

\footnotetext{
${ }^{2}$ We show in (3.4) that $\lambda_{\sigma} / \lambda_{\sigma}^{\prime} \in \mathbb{Q}$ for $\sigma \in \Sigma_{\mathfrak{a}}$.
} 
Similarly, let $h(D)$ be the number of primitive quadratic forms of discriminant $D<0$, and set

$$
h_{0}(D)=\frac{2 h(D)}{w(D)}
$$

where $w(D)$ is the number of units of the quadratic order of discriminant $D$. We extend the definition to all $D$ by setting $h_{0}(0)=-1 / 12, h_{0}\left(u^{2}\right)=-\varphi(u) / 2$ if $u>0$ is an integer, and $h_{0}(D)=0$ if $D$ is not a negative discriminant or a square. This extension of $h_{0}(D)$ is compatible to that of $H(D)$, in the sense that for all $D$ we have

$$
H(-D)=\sum_{d^{2} \mid D} h_{0}\left(\frac{D}{d^{2}}\right), \quad h_{0}(-D)=\sum_{d^{2} \mid D} H\left(\frac{D}{d^{2}}\right) \mu(d),
$$

with the understanding that for $D=0$ only $d=1$ is included in both sums. It follows that for all $D \neq 0$ and for $u \geqslant 1$ we have

$$
\sum_{\substack{X \subset \overline{\mathcal{M}}_{n} \\
\Delta(X)=D, G_{X}=u}} \varepsilon(X)=\left\{\begin{array}{ll}
-2 h_{0}\left(D / u^{2}\right) & \text { if } \operatorname{Tr}(X) \neq 0 \\
-h_{0}\left(D / u^{2}\right) & \text { if } \operatorname{Tr} X=0
\end{array},\right.
$$

and with the convention that $0 / 0^{2}=0$ this formula also holds for $D=0$ and $u=0$.

The class numbers enter the trace formula under the following assumption on the function $\mathcal{C}_{\Gamma, \Sigma}^{\chi}$ in Theorems 1 and 2. Let $G_{M}=\operatorname{gcd}(c, d-a, b)$ be the content of the quadratic form $Q_{M}$ associated to the matrix $M$.

Assumption 2.1. If $\Gamma$ is a congruence subgroup of level $N$, then the function $\mathcal{C}_{\Gamma, \Sigma}^{\chi}(M)$ in Theorem 1 only depends on the conjugacy class invariants $\operatorname{Tr} M$, $\operatorname{det} M$, and $\left(N, G_{M}\right)$, namely there is an arithmetic function $B_{\Gamma, \Sigma}^{\chi}(u, t, n)$ such that

$$
\mathcal{C}_{\Gamma, \Sigma}^{\chi}(M)=B_{\Gamma, \Sigma}^{\chi}\left(\left(N, G_{M}\right), \operatorname{Tr} M, \operatorname{det} M\right), \quad \text { for all } M \in \mathcal{M} .
$$

The assumption is natural, as the function $\mathcal{C}_{\Gamma, \Sigma}^{\chi}$ is constant on $\Gamma_{1}$-conjugacy classes in $\mathcal{M}$, and we will see that it is satisfied for the double cosets giving the action of the usual Hecke and Atkin-Lehner operators for $\Gamma=\Gamma_{0}(N)$.

Moebius inversion gives a function $C_{\Gamma, \Sigma}^{\chi}(u, t, n)$ such that

$$
B_{\Gamma, \Sigma}^{\chi}(u, t, n)=\sum_{d \mid u} C_{\Gamma, \Sigma}^{\chi}(d, t, n), \quad C_{\Gamma, \Sigma}^{\chi}(u, t, n)=\sum_{d \mid u} B_{\Gamma, \Sigma}^{\chi}(u / d, t, n) \mu(d),
$$

where we assume $u \mid N$ in both formulas. Note that the functions $B_{\Gamma, \Sigma}^{\chi}, C_{\Gamma, \Sigma}^{\chi}$ are only defined on triples $(u, t, n)$ with $u\left|N, u^{2}\right| t^{2}-4 n$, and they scale by $(-1)^{k}$ when $t$ is replaced by $-t$.

Let $\Sigma \subset \mathcal{M}_{n}$ be a double coset, with $\mathcal{M}_{n} \subset \mathcal{M}$ consisting of matrices of determinant $n$. Under Assumption 2.1, the sum over $\Gamma_{1}$-conjugacy classes

$$
\text { RHS }:=\sum_{X \subset \overline{\mathcal{M}}_{n}} p_{k-2}\left(\operatorname{Tr} M_{X}, n\right) \mathcal{C}_{\Gamma, \Sigma}^{\chi}\left(M_{X}\right) \varepsilon(X)
$$


in the right hand side of $(2.5)$ becomes $^{3}$

$$
\begin{aligned}
\text { RHS } & =\sum_{t \in \mathbb{Z} /\{ \pm 1\}} p_{k-2}(t, n) \sum_{u \mid N} C_{\Gamma, \Sigma}^{\chi}(u, t, n) \sum_{\substack{X \subset \overline{\mathcal{M}}_{n} \\
\operatorname{Tr}(X)=t, u \mid G_{X}}} \varepsilon(X) \\
& =-\sum_{t \in \mathbb{Z}} p_{k-2}(t, n) \sum_{u \mid N} H\left(\frac{4 n-t^{2}}{u^{2}}\right) C_{\Gamma, \Sigma}^{\chi}(u, t, n) .
\end{aligned}
$$

In the second equality we used (2.10), and the fact that $C_{\Gamma, \Sigma}^{\chi}$ scales by $(-1)^{k}$ when $t$ is replaced by $-t$, just like $p_{k-2}(t, n)$.

Similarly using the extended class numbers $h_{0}(D)$ from $(2.12)$ we have

$$
\begin{aligned}
\mathrm{RHS} & =\sum_{t \in \mathbb{Z} /\{ \pm 1\}} p_{k-2}(t, n) \sum_{u^{2} \mid t^{2}-4 n}^{\prime} B_{\Gamma, \Sigma}^{\chi}((N, u), t, n) \sum_{\substack{X \subset \overline{\mathcal{M}}_{n} \\
\operatorname{Tr}(X)=t, G_{X}=u}} \varepsilon(X) \\
& =-\sum_{t \in \mathbb{Z}} p_{k-2}(t, n) \sum_{u} h_{0}\left(\frac{t^{2}-4 n}{u^{2}}\right) B_{\Gamma, \Sigma}^{\chi}((N, u), t, n),
\end{aligned}
$$

with the understading that only $u=N$ is to be considered in the summation if $t^{2}-4 n=0$. Note that if we restrict the sum over $X$ in (2.14) to elliptic conjugacy classes, the range of summation in $t$ in the last terms of (2.15) and (2.16) becomes $t^{2}<4 n$. Thus we obtain two explicit expressions in terms of class numbers of the right hand side of (2.7).

Remark 2.2. The case $k=2$ of the trace formula reduces to the Kronecker-Hurwitz class number formula. Indeed, for $k=2, \Gamma=\Gamma_{1}, \widetilde{\chi}=1$, and $\Sigma=\mathcal{M}_{n}$, the left side of (2.5) vanishes, and using (2.15) we obtain the Kronecker-Hurwitz relation: $\sum_{t \in \mathbb{Z}} H\left(4 n-t^{2}\right)=\sigma_{1}(n)$. Taking $\Sigma$ to be any $\Gamma_{1}$-double coset instead of $\mathcal{M}_{n}$, the case $k=2, \Gamma=\Gamma_{1}$ of the trace formula (2.5) gives a group-theoretical way of writing the Kronecker-Hurwitz formula in terms of the invariants $\varepsilon(X)$ :

$$
\sum_{X \subset \bar{\Sigma}} \varepsilon(X)=-\left|\Gamma_{1} \backslash \Sigma\right|
$$

with the sum over $\bar{\Gamma}_{1}$-conjugacy classes in $\bar{\Sigma}$. Together with D. Zagier, we give an elementary proof of a refinement of this formula in [9].

2.3. Trace formulas on $\Gamma_{0}(N)$ and $\Gamma_{1}(N)$. We now specialize $\Gamma=\Gamma_{0}(N)$. Let $k \geqslant 2, \chi$ a character modulo $N$ with $\chi(-1)=(-1)^{k}$, and let $\Sigma=\Delta_{n}$ be the double coset of the usual Hecke operator acting on $S_{k}(N, \chi):=S_{k}(\Gamma, \chi)$, given in $(2.3)$. Changing notation $\mathcal{C}_{N, \chi}(M)=\mathcal{C}_{\Gamma, \Sigma}^{\chi}(M)$, we have

$$
\mathcal{C}_{N, \chi}(M)=\sum_{\substack{A \in \Gamma \backslash \Gamma_{1} \\ A M A^{-1} \in \Delta_{n}}} \chi\left(a_{A M A^{-1}}\right)
$$

\footnotetext{
${ }^{3}$ In all formulas in this paper we adopt the convention that arithmetic functions are zero on nonintegers. For example the sums over $u$ in (2.15) and (2.16) are restricted to $u^{2} \mid t^{2}-4 n$.
} 
This function was computed by Oesterlé [6] (Lemma 4.1 below), who showed that it only depends on the conjugacy class invariants $t=\operatorname{Tr} M, n=\operatorname{det} M$, and $u=\left(G_{M}, N\right)$ :

$$
\mathcal{C}_{N, \chi}(M)=B_{N, \chi}(u, t, n):=\frac{\varphi_{1}(N)}{\varphi_{1}(N / u)} \sum_{x \in S_{N}(u, t, n)} \chi(x),
$$

where $S_{N}(u, t, n)=\left\{\alpha \in(\mathbb{Z} / N \mathbb{Z})^{\times}: \alpha^{2}-t \alpha+n \equiv 0(\bmod N u)\right\},{ }^{4}$ and $\varphi_{1}(N)$ is the index of $\Gamma_{0}(N)$ in $\Gamma_{1}$, equal to $N \prod_{p \mid N}(1+1 / p)$. Notice that $B_{N, \chi}(u, t, n)$ is multiplicative in $N$, so its Moebius inverse

$$
C_{N, \chi}(u, t, n):=\sum_{d \mid u} B_{N, \chi}(u / d, t, n) \mu(d)
$$

is also multiplicative, and it can be easily computed numerically.

We can now state the trace formula on the cuspidal subspace, which follows from Theorem 2 after expressing the right hand side as in (2.15), and using the formula for the cuspidal sum $\Phi_{N, \chi}(a, d)$ proved in $\S 4.1$. Note that the simpler formula for the linear combination (1.1), stated in the introduction, holds also for characters $\chi$ with $\chi(-1) \neq(-1)^{k}$, when both sides vanish.

Theorem 3. Let $N \geqslant 1$ and $k \geqslant 2$ be integers, and $\chi$ a character $\bmod N$ with $\chi(-1)=(-1)^{k}$. With the function $C_{N, \chi}(u, t, n)$ defined above, we have

$$
\begin{aligned}
\operatorname{Tr}\left(T_{n}, S_{k}(N, \chi)\right)= & -\frac{1}{2} \sum_{t^{2} \leqslant 4 n} p_{k-2}(t, n) \cdot \sum_{u \mid N} H\left(\frac{4 n-t^{2}}{u^{2}}\right) C_{N, \chi}(u, t, n) \\
& -\frac{1}{2} \sum_{n=a d} \min (a, d)^{k-1} \Phi_{N, \chi}(a, d)+\delta_{k, 2} \delta_{\chi, \mathbf{1}} \sigma_{1, N}(n),
\end{aligned}
$$

where $\sigma_{1, N}(n)=\sum_{d \mid n,(N, d)=1} n / d$, and

$$
\Phi_{N, \chi}(a, d)=\sum_{\substack{N=r s \\(r, s) \mid(N / c(\chi), a-d)}} \varphi((r, s)) \chi\left(\alpha_{r, s}^{a, d}\right),
$$

where $\alpha=\alpha_{r, s}^{a, d}$ is the residue class modulo $N /(r, s)$ such that $\alpha \equiv a(\bmod r), \alpha \equiv d(\bmod s), c(\chi)$ is the conductor of $\chi$, and $\varphi$ denotes Euler's function.

The terms for $t^{2}=4 n$ are explictly computed in Remark 3.8: they are nonzero only when $n$ is a square, when they contribute $\frac{\varphi_{1}(N)}{12}(k-1) n^{k / 2-1} \chi(\sqrt{n})$.

Remark. An equivalent formula for the cuspidal trace was obtained by Cohen and Oesterlé by analytic means $[2,6]$, with the sum over $u$ written as in (2.16) in terms of $B_{N, \chi}$.

We also give a formula for the trace of $T_{n} \circ W_{\ell}$ on $S_{k}(N)$, with $W_{\ell}$ the Atkin-Lehner operator for an exact divisor $\ell$ of $N$. Let $C_{N}(u, t, n)=C_{N, \mathbf{1}}(u, t, n)$ in (2.18), with 1 the trivial character $\bmod N$. A different formula for $\operatorname{Tr}\left(T_{n} \circ W_{\ell}, S_{k}(N)\right)$ was given by Skoruppa and Zagier [16], but assuming that $(n, N)=1$.

\footnotetext{
${ }^{4}$ This set is well defined: if $\alpha \in \mathbb{Z}$ satisfies $\alpha^{2}-t \alpha+n \equiv 0(\bmod N u)$, so does $\alpha+N d$ for every $d$, because of the assumption $u\left|N, u^{2}\right| t^{2}-4 n$.
} 
Theorem 4. Let $N=\ell \ell^{\prime}$ with $\left(\ell, \ell^{\prime}\right)=1$ and $k \geqslant 2$ even, $w=k-2$. For all $n \geqslant 1$ we have

$$
\begin{array}{r}
\operatorname{Tr}\left(T_{n} \circ W_{\ell}, S_{k}(N)\right)=-\frac{1}{2} \sum_{\substack{t^{2} \leqslant 4 \ell n \\
\ell \mid t}} \frac{p_{w}(t, \ell n)}{\ell^{w / 2}} \cdot \sum_{\begin{array}{c}
u \mid \ell \\
u^{\prime} \mid \ell^{\prime}
\end{array}} H\left(\frac{4 \ell n-t^{2}}{\left(u u^{\prime}\right)^{2}}\right) C_{\ell^{\prime}}\left(u^{\prime}, t, \ell n\right) \mu(u) \\
-\frac{1}{2} \sum_{\begin{array}{c}
n \ell=a d \\
\ell \mid a+d
\end{array}} \frac{\min (a, d)^{k-1}}{\ell^{w / 2}} \Phi_{N, \ell}(a, d)+\delta_{k, 2} \sigma_{1, N}(n),
\end{array}
$$

where

$$
\Phi_{N, \ell}(a, d)=\frac{\varphi(\ell)}{\ell} \sum_{\substack{\ell^{\prime}=r s,(r, s) \mid a-d \\(r, a)=1,(s, d)=1}} \varphi((r, s)) .
$$

The terms for $t^{2}=4 \ell n$ in the summation above are present only if $\ell=1, n$ is a square, and $(n, N)=1$, when they contribute $\frac{\varphi_{1}(N)}{12}(k-1) n^{w / 2}$. Note that $\Phi_{N, 1}$ is the same as the function $\Phi_{N, \chi}$ in Theorem 3 for $\chi=\mathbf{1}$.

The function $C_{N}(u, t, n)$ is explicitly computed in Lemma 4.5, where we show that

$$
C_{N}(u, t, n)=\left|S_{N}(t, n)\right| \cdot C_{N}\left(u, t^{2}-4 n\right),
$$

with $S_{N}(t, n)=\left\{\alpha \in(\mathbb{Z} / N \mathbb{Z})^{\times}: \alpha^{2}-t \alpha+n \equiv 0(\bmod N)\right\}$, and $C_{N}(u, D)$ an explicit multiplicative function in $(N, u)$. For example, when $N$ is square-free, we have $C_{N}(u, D)=u$ independent of $D$.

We also obtain a trace formula for $\Gamma_{1}(N)$, by summing over $\chi$ the formula in Theorem 3 . For $u\left|N, u^{2}\right| t^{2}-4 n$ we let

$$
B_{N}(u, t, n)=\frac{1}{\varphi(N)} \sum_{\chi} B_{N, \chi}(u, t, n)=\left\{\begin{array}{cl}
\frac{\varphi_{1}(N)}{\varphi_{1}(N / u)} & \text { if } N u \mid t-n-1 \\
0 & \text { otherwise }
\end{array}\right.
$$

where the sum is over all the $\varphi(N)$ characters modulo $N$. Let $D_{N}(u, t, n)$ denote its Moebius inverse defined as in (2.18).

Theorem 5. Let $N \geqslant 1, k \geqslant 2, n \geqslant 1$, and set $\Gamma=\Gamma_{1}(N)$. We have:

$$
\begin{gathered}
\operatorname{Tr}\left(T_{n}, S_{k}(\Gamma)+M_{k}(\Gamma)\right)=\delta_{k, 2} \sigma_{1, N}(n)-\varphi(N) \cdot \sum_{\substack{t \in \mathbb{Z} \\
N \mid t-n-1}} p_{k-2}(t, n) \cdot \sum_{u \mid N} H\left(\frac{4 n-t^{2}}{u^{2}}\right) D_{N}(u, t, n), \\
\operatorname{Tr}\left(T_{n}, S_{k}(\Gamma)\right)=\delta_{k, 2} \sigma_{1, N}(n)-\frac{\varphi(N)}{2} \cdot \sum_{t^{2} \leqslant 4 n} p_{k-2}(t, n) \cdot \sum_{u \mid N} H\left(\frac{4 n-t^{2}}{u^{2}}\right) D_{N}(u, t, n) \\
-\frac{1}{4} \sum_{n=a d} \min (a, d)^{k-1}\left(\Psi_{N}(a, d)+(-1)^{k} \Psi_{N}(-a,-d)\right)
\end{gathered}
$$

where

$$
\Psi_{N}(a, d)=\sum_{\substack{N=r s \\ r|(a-1), s|(d-1)}} \varphi((r, s)) \varphi(N /(r, s)) .
$$

The formulas in Theorems 3, 4 and 5 were verified numerically for a large range of parameters. 
2.4. Applications. An important application of the trace formula is that it can be used to construct explicitly modular forms. Indeed the "trace form"

$$
\sum_{n \geqslant 1} \operatorname{Tr}\left(T_{n}, S_{k}(N, \chi)\right) e^{2 \pi i n z},
$$

is a nonzero modular form belonging to $S_{k}(N, \chi)$, and by acting on it with Hecke operators one can generate the entire space.

We give two explicit examples for $N=4$. In this case it is easier to apply Theorem 5 , since $\operatorname{Tr}\left(T_{n}, S_{k}\left(\Gamma_{1}(4)\right)\right.$ equals the trace on $S_{k}(4)$ for $k$ even, and the trace on $S_{k}\left(4, \chi_{4}\right)$ for $k$ odd, with $\chi_{4}$ the nontrivial character modulo 4 . The functions $D_{N}(u, t, n)$ in Theorem 5 are straightforward to compute, and we obtain the following explicit formulas. More details are given in Section 4.3.

Corollary 1. a) Let $n \geqslant 1$ be odd. If $k \geqslant 2$ is even we have

$$
\operatorname{Tr}\left(T_{n}, S_{k}(4)\right)=-3 \sum_{s^{2} \leqslant n} p_{k-2}(2 s, n) H\left(n-s^{2}\right)-\frac{3}{2} \sum_{n=a d} \min (a, d)^{k-1}+\delta_{k, 2} \sigma_{1}(n),
$$

while if $k \geqslant 3$ is odd we have

$$
\begin{gathered}
\operatorname{Tr}\left(T_{n}, S_{k}\left(4, \chi_{4}\right)\right)=-\sum_{\substack{s^{2} \leqslant n \\
s \text { odd }}}(-1)^{(2 s-n-1) / 4} p_{k-2}(2 s, n)\left(H\left(n-s^{2}\right)+2 H\left(\frac{n-s^{2}}{4}\right)\right) \\
-\sum_{\substack{n=a d \\
4 \mid a-d}} \min (a, d)^{k-1} \chi_{4}(a) .
\end{gathered}
$$

b) Let $n \geqslant 2$ be even. For $k \geqslant 2$, and $\chi$ the character $\bmod 4$ with $\chi(-1)=(-1)^{k}$ we have

$$
\operatorname{Tr}\left(T_{n}, S_{k}(4, \chi)\right)=-\sum_{\substack{t^{2} \leqslant 4 n \\
4 \mid t-n-1}} p_{k-2}(t, n) H\left(4 n-t^{2}\right)-\sum_{\substack{n=a d \\
a \text { odd }}} \chi(a) \min (a, d)^{k-1}+\delta_{k, 2} \sum_{\begin{array}{c}
n=a d \\
a \text { odd }
\end{array}} d .
$$

Remark. Note that in both sums over $s$ in part a) we have $4 \mid 2 s-n-1$ in order for $H\left(n-s^{2}\right) \neq 0$. Note that $\operatorname{Tr}\left(T_{n}, S_{k}\left(4, \chi_{4}\right)\right)=0$ for $n \equiv 3(\bmod 4)$, as all terms in the formula vanish.

For $f=\sum_{n \geqslant 1} a_{n} q^{n}$ a modular form in $S_{k}(4)$, respectively in $S_{k}\left(4, \chi_{4}\right)$ it is easy to see that both $\sum_{n \text { odd }} a_{n} q^{n}$ and $\sum_{n \text { even }} a_{n} q^{n}$ belong to $S_{k}(4)$, respectively to $S_{k}\left(8, \chi_{4}\right)$. Applying this observation to the trace form, we obtain explicit cusp forms of the type given in the introduction. For $k$ even and odd index traces, this proves a conjecture of Cohen [1], which was recently proved by Mertens by different methods [5]. For $k$ odd, and for $k$ even and even index traces, the resulting formulas seem to be new.

Since the spaces $S_{2}(4), S_{3}\left(4, \chi_{4}\right)$ are trivial, the formulas in the corollary reduce to class number relations similar to the Kronecker-Hurwitz formula. Other such relations can be obtained from Theorem 4 taking small values for $N$.

As a second application, we use Theorem 5 to obtain the limit stated in the introduction for the trace of a fixed Hecke operator $T_{n}$ on $\Gamma_{1}(N)$ when $N$ goes to infinity. In fact we obtain precise formulas for $N>2 n+2$, which no longer contain class numbers: 
Corollary 2. Fix $k \geqslant 2$ and $n>1$. For $N>2 n+2$ we have:

$$
\begin{gathered}
\operatorname{Tr}\left(T_{n}, S_{k}\left(\Gamma_{1}(N)\right)+M_{k}\left(\Gamma_{1}(N)\right)\right)=\delta_{k, 2} \sigma_{1, N}(n)+\frac{\varphi(N)}{2} \frac{n^{k-1}-1}{n-1} \sum_{u \mid n-1} \varphi(u) \frac{\varphi_{1}(N)}{\varphi_{1}(N /(u, N))} \\
\operatorname{Tr}\left(T_{n}, S_{k}\left(\Gamma_{1}(N)\right)\right)=\delta_{k, 2} \sigma_{1, N}(n)-\frac{1}{2} \sum_{u \mid(N, n-1)} \varphi((u, N / u)) \varphi\left(\frac{N}{(u, N / u)}\right) .
\end{gathered}
$$

Assuming $(N, n-1)=1$, the first formula gives:

$$
\lim _{\substack{N \rightarrow \infty \\(N, n-1)=1}} \frac{\operatorname{Tr}\left(T_{n}, S_{k}\left(\Gamma_{1}(N)\right)+M_{k}\left(\Gamma_{1}(N)\right)\right)}{\varphi(N)}=\frac{n^{k-1}-1}{2},
$$

while the second gives the limit formula from in the introduction.

Proof. Since $N \mid t-n-1$ in the sums in Theorem 5, the assumption $N>2 n+2$ implies that only $t=n+1$ contributes in the first formula and the sum over $t$ in the second formula is empty. We also have $\Phi_{N}(-a,-d)=0$ for $n=a d, a, d>0$, and since $p_{k-2}(n+1, n)=\left(n^{k-1}-1\right) /(n-1)$ the conclusion follows.

\section{General trace formulas on the Eisenstein subspace and on the Cuspidal SUBSPACE}

In Section 3.1 we take $\Gamma$ to be Fuchsian subgroup of the first kind with cusps and we compute the trace of a double coset operator $[\Sigma]$ on the Eisenstein subspace $E_{k}(\Gamma, \chi)$. Here $\Sigma$ is any double coset contained in the commensurator of $\Gamma$ inside $\mathrm{GL}_{2}^{+}(\mathbb{R})$. As an immediate consequence, in Section 3.2 we use an equivalent formulation of Theorem 1 that makes sense for a Fuchsian group (see (3.17)) to obtain the trace formula on the cuspidal subspace in Theorem 2.

The trace formulas on the Eisenstein and on the cuspidal subspaces depend on the arithmetic function $\Phi_{\Gamma, \Sigma}^{\chi}(a, d)$, and in Section 3.3 we give a practical way to compute this function when $\Gamma$ is a finite index subgroup of $\Gamma_{1}$ and $\Sigma$ is a double coset satisfying the assumption in Theorem 1 , which we restate here as follows.

Assumption 3.1. The map

$$
\Gamma \backslash \Sigma \longrightarrow \Gamma_{1} \backslash \Gamma_{1} \Sigma, \quad \Gamma \sigma \mapsto \Gamma_{1} \sigma
$$

is bijective, or equivalently $|\Gamma \backslash \Sigma|=\left|\Gamma_{1} \backslash \Gamma_{1} \Sigma\right|$.

3.1. A trace formula on the Eisenstein subspace. We start by introducing some notation and terminology related to the cusps. For a parabolic or hyperbolic matrix $\sigma \in \mathrm{GL}_{2}(\mathbb{R})^{+}$, we denote by $\operatorname{sgn}(\sigma) \in\{ \pm 1\}$ the sign of the eigenvalues of $\sigma$. For $\mathfrak{a}$ a cusp of $\Gamma$, we let $\Gamma_{\mathfrak{a}} \subset \Gamma$ be the stabilizer of $\mathfrak{a}$ in $\Gamma$. Thus $\Gamma_{\mathfrak{a}}= \pm\left\langle\gamma_{\mathfrak{a}}\right\rangle$ if $-1 \in \Gamma$, and $\Gamma_{\mathfrak{a}}=\left\langle\gamma_{\mathfrak{a}}\right\rangle$ if $-1 \notin \Gamma$, for a generator $\gamma_{\mathfrak{a}} \in \Gamma_{\mathfrak{a}}$, with $\operatorname{sgn}\left(\gamma_{\mathfrak{a}}\right)=+1$ if $-1 \in \Gamma$. Let $C_{\mathfrak{a}} \in \mathrm{SL}_{2}(\mathbb{R})$ be a scaling matrix for the cusp $\mathfrak{a}$, namely $C_{\mathfrak{a}} \mathfrak{a}=\infty$, and

$$
C_{\mathfrak{a}} \gamma_{\mathfrak{a}} C_{\mathfrak{a}}^{-1}=\operatorname{sgn}\left(\gamma_{\mathfrak{a}}\right)\left(\begin{array}{ll}
1 & 1 \\
0 & 1
\end{array}\right) .
$$

We assume that scaling matrices for equivalent cusps satisfy $C_{\gamma \mathfrak{a}}=C_{\mathfrak{a}} \gamma^{-1}$. 
For $\sigma \in \widetilde{\Gamma}$ (the commensurator of $\Gamma$ ), there exists $n \in \mathbb{Z}$ such that $\gamma_{\mathfrak{a}}^{n} \in \sigma^{-1} \Gamma \sigma$, and since $\sigma \gamma_{\mathfrak{a}}^{n} \sigma^{-1} \in \Gamma$ is a parabolic element fixing $\sigma \mathfrak{a}$, we have that $\mathfrak{b}=\sigma \mathfrak{a}$ is also a cusp of $\Gamma$, and

$$
\sigma \gamma_{\mathfrak{a}}^{n} \sigma^{-1}= \pm \gamma_{\mathfrak{b}}^{m}
$$

for some $m \in \mathbb{Z}$. As $C_{\mathfrak{b}} \sigma C_{\mathfrak{a}}^{-1} \infty=\infty$ we have

$$
C_{\mathfrak{b}} \sigma C_{\mathfrak{a}}^{-1}=\left(\begin{array}{cc}
a_{\mathfrak{a}}(\sigma) & b \\
0 & d_{\mathfrak{a}}(\sigma)
\end{array}\right)
$$

for some $a_{\mathfrak{a}}(\sigma), d_{\mathfrak{a}}(\sigma) \in \mathbb{R}$, and therefore $C_{\mathfrak{b}} \sigma \gamma_{\mathfrak{a}} \sigma^{-1} C_{\mathfrak{b}}^{-1}= \pm\left(\begin{array}{cc}1 & a_{\mathfrak{a}}(\sigma) / d_{\mathfrak{a}}(\sigma) \\ 0 & 1\end{array}\right)$. Raising the previous relation to the power $2 n$ and using (3.2) we obtain that the ratio

$$
\frac{a_{\mathfrak{a}}(\sigma)}{d_{\mathfrak{a}}(\sigma)}=\frac{m}{n}
$$

is a positive rational number.

Remark 3.2. For fixed $\mathfrak{a}$, the constants $a_{\mathfrak{a}}(\sigma), d_{\mathfrak{a}}(\sigma)$ only depend on the coset $\Gamma \sigma$ since $C_{\gamma \mathfrak{b}}=$ $C_{\mathfrak{b}} \gamma^{-1}$. Moreover $a_{\mathfrak{a}}(\sigma), d_{\mathfrak{a}}(\sigma)$ are invariant under the map $(\mathfrak{a}, \sigma) \mapsto\left(\gamma \mathfrak{a}, \sigma \gamma^{-1}\right)$, for $\gamma \in \Gamma$. It follows that by scaling the double coset $\Sigma$ we can assume that $a_{\mathfrak{a}}(\sigma), d_{\mathfrak{a}}(\sigma) \in \mathbb{Z}$ for all cusps $\mathfrak{a}$ and $\sigma \in \Sigma$.

3.1.1. Constant terms of Eisenstein series. For an Eisenstein series $E \in E_{k}(\Gamma, \chi)$, the constant term $A_{E}(\mathfrak{a})$ of $E$ at the cusp $\mathfrak{a}$ is defined as the constant term of the Fourier expansion of $\left.E\right|_{k} C_{\mathfrak{a}}^{-1}$ :

$$
A_{E}(\mathfrak{a})=a_{0}\left(\left.E\right|_{k} C_{\mathfrak{a}}^{-1}\right)=\left.\lim _{z \rightarrow i \infty} E\right|_{k} C_{\mathfrak{a}}^{-1}(z) .
$$

From (3.1) we have $A_{E}(\mathfrak{a})=a_{0}\left(\left.E\right|_{k} C_{\mathfrak{a}}^{-1} T^{-1}\right)=\chi\left(\gamma_{\mathfrak{a}}\right)^{-1} \operatorname{sgn}\left(\gamma_{\mathfrak{a}}\right)^{k} a_{0}\left(\left.E\right|_{k} C_{\mathfrak{a}}^{-1}\right)$, so $A_{E}(\mathfrak{a})$ vanishes unless $\chi\left(\gamma_{\mathfrak{a}}\right)=\operatorname{sgn}\left(\gamma_{\mathfrak{a}}\right)^{k}$. Therefore we define the $\Gamma$-invariant set

$$
\operatorname{Cusps}(\Gamma, \chi)=\left\{\mathfrak{a} \in \operatorname{Cusps}(\Gamma): \chi(\gamma)=\operatorname{sgn}(\gamma)^{k} \text { for } \gamma \in \Gamma_{\mathfrak{a}}\right\},
$$

and we let $C(\Gamma, \chi) \subset C(\Gamma)$ be sets of representatives for $\Gamma$-equivalence classes in $\operatorname{Cusps}(\Gamma, \chi)$, respectively in $\operatorname{Cusps}(\Gamma)$. When $\chi=\mathbf{1}$, the trivial character, we have $C(\Gamma, \mathbf{1})=C(\Gamma)$ if $k$ is even, while $C(\Gamma, \mathbf{1})$ is the set of regular cusps if $k$ is odd and $-1 \notin \Gamma$.

Since $C_{\mathfrak{a}} \gamma^{-1}$ is a scaling matrix for $\gamma \mathfrak{a}$ for $\gamma \in \Gamma$, it follows that $A_{E}(\gamma \mathfrak{a})=\chi(\gamma) A_{E}(\mathfrak{a})$. Identifying the vector space $\mathbb{C}^{|C(\Gamma, \chi)|}$ with the space of maps $f: C(\Gamma, \chi) \rightarrow \mathbb{C}$, we have an injective map

$$
E_{k}(\Gamma, \chi) \longrightarrow \mathbb{C}^{|C(\Gamma, \chi)|}, \quad E \mapsto A_{E} .
$$

This map is a bijection, unless $k=2$ and $\chi=\mathbf{1}$, when $C(\Gamma, \mathbf{1})=C(\Gamma)$ and we have an exact sequence

$$
0 \longrightarrow E_{2}(\Gamma) \stackrel{E \mapsto A_{E}}{\longrightarrow} \mathbb{C}^{|C(\Gamma)|} \stackrel{f \mapsto \sum_{\mathfrak{a}} f(\mathfrak{a})}{\longrightarrow} \mathbb{C} \longrightarrow 0 .
$$

We can now compute the constant terms of $E \mid[\Sigma]$, for $\Sigma \subset \widetilde{\Gamma}$ a double coset. For a cusp $\mathfrak{a} \in \operatorname{Cusps}(\Gamma, \chi)$ and $E \in E_{k}(\Gamma, \chi)$ we have by $(2.2)$

$$
E|[\Sigma]|_{k} C_{\mathfrak{a}}^{-1}=\left.\sum_{\sigma \in \Gamma \backslash \Sigma} \operatorname{det} \sigma^{k-1} \tilde{\chi}(\sigma) E\right|_{k} \sigma C_{a}^{-1} .
$$


Replacing $\sigma C_{\mathfrak{a}}^{-1}$ from (3.3) in the previous relation, and taking $z \rightarrow i \infty$ we obtain

$$
a_{0}\left(E|[\Sigma]|_{k} C_{\mathfrak{a}}^{-1}\right)=\sum_{\sigma \in \Gamma \backslash \Sigma} a_{0}\left(\left.E\right|_{k} C_{\sigma \mathfrak{a}}^{-1}\right) \frac{a_{\mathfrak{a}}(\sigma)^{k-1}}{d_{\mathfrak{a}}(\sigma)} \tilde{\chi}(\sigma) .
$$

3.1.2. The trace formula. Using (3.7), we compute $\operatorname{Tr}\left([\Sigma], E_{k}(\Gamma, \chi)\right)$ in the next theorem. First we prove a lemma interesting in its own right, which is needed for the case $k=2, \chi=\mathbf{1}$, and whose proof will be used in the proof of the theorem. We make the following assumption on the double coset $\Sigma \subset \widetilde{\chi}$, which is implied but much weaker than assumption 3.1.

Assumption 3.3. If $-1 \notin \Gamma$, then $\Sigma \cap-\Sigma=\emptyset$.

Lemma 3.4. Let $\Gamma$ be a Fuchsian group of the first kind, and let $\Sigma \subset \widetilde{\Gamma}$ be a double coset satisfying Assumption 3.3. Then

$$
\sum_{\sigma \in \Gamma \backslash \Sigma} \frac{a_{\mathfrak{a}}(\sigma)}{d_{\mathfrak{a}}(\sigma)}=|\Gamma \backslash \Sigma|,
$$

independent of the cusp $\mathfrak{a}$ of $\Gamma$, where $C_{\sigma \mathfrak{a}} \sigma C_{\mathfrak{a}}^{-1}=\left(\begin{array}{cc}a_{\mathfrak{a}}(\sigma) & * \\ 0 & d_{\mathfrak{a}}(\sigma)\end{array}\right)$.

Proof. For each $\mathfrak{b} \in C(\Gamma)$, let $\Sigma_{\mathfrak{a} \mathfrak{b}}:=\{\sigma \in \Sigma: \sigma \mathfrak{a}=\mathfrak{b}\}$. Each coset $\Gamma \sigma$ contains a representative $\sigma_{0}$ with $\sigma_{0} \mathfrak{a}=\mathfrak{b}$, where $\mathfrak{b}$ is the fixed representative in $C(\Gamma)$ of the equivalence class of cusps $\Gamma \sigma \mathfrak{a}$, and if $\gamma \sigma_{0}$ is another such representative we have $\gamma \in \Gamma_{\mathfrak{b}}$. A similar reasoning applies to right cosets, so we have the disjoint decompositions (with a slight abuse of notation)

$$
\Gamma \backslash \Sigma=\bigcup_{\mathfrak{b} \in C(\Gamma)} \Gamma_{\mathfrak{b}} \backslash \Sigma_{\mathfrak{a} \mathfrak{b}}, \quad \Sigma / \Gamma=\bigcup_{\mathfrak{b} \in C(\Gamma)} \Sigma_{\mathfrak{a} \mathfrak{b}} / \Gamma_{\mathfrak{a}}
$$

For $a, d>0$, let

$$
\Sigma_{\mathfrak{a} \mathfrak{b}}(a, d)=\left\{\sigma \in \Sigma_{\mathfrak{a} \mathfrak{b}}: C_{\mathfrak{b}} \sigma C_{\mathfrak{a}}^{-1}=\operatorname{sgn}(\sigma)\left(\begin{array}{ll}
a & * \\
0 & d
\end{array}\right)\right\}=\Sigma_{\mathfrak{a} \mathfrak{b}}^{+}(a, d) \cup \Sigma_{\mathfrak{a} \mathfrak{b}}^{-}(a, d),
$$

where $\Sigma_{\mathfrak{a} \mathfrak{b}}^{ \pm}(a, d)$ consist of those $\sigma \in \Sigma_{\mathfrak{a} \mathfrak{b}}(a, d)$ having $\operatorname{sgn}(\sigma)= \pm 1$. The first decomposition gives

$$
\sum_{\sigma \in \Gamma \backslash \Sigma} \frac{a_{\mathfrak{a}}(\sigma)}{d_{\mathfrak{a}}(\sigma)}=\sum_{a, d>0} a \sum_{\mathfrak{b} \in C(\Gamma)} \frac{1}{d} \cdot\left|\Gamma_{\mathfrak{b}} \backslash \Sigma_{\mathfrak{a} \mathfrak{b}}(a, d)\right| .
$$

The set $\Sigma_{\mathfrak{a} \mathfrak{b}}(a, d)$ is left invariant by $\Gamma_{\mathfrak{b}}$ and right invariant by $\Gamma_{\mathfrak{a}}$, and we show that

$$
\frac{1}{d} \cdot\left|\Gamma_{\mathfrak{b}} \backslash \Sigma_{\mathfrak{a} \mathfrak{b}}(a, d)\right|=\frac{1}{(a, d)} \cdot\left|\Gamma_{\mathfrak{b}} \backslash \Sigma_{\mathfrak{a} \mathfrak{b}}(a, d) / \Gamma_{\mathfrak{a}}\right|=\frac{1}{a} \cdot\left|\Sigma_{\mathfrak{a} \mathfrak{b}}(a, d) / \Gamma_{\mathfrak{a}}\right| .
$$

To prove this identity, we assume by Remark 3.2 that $a, d \in \mathbb{Z}$. We also assume for simplicity that $-1 \in \Gamma$, the other case being similar (using Assumption 3.3). We have $\Gamma_{\mathfrak{b}} \backslash \Sigma_{\mathfrak{a b}}(a, d)=$ $\left\langle\gamma_{\mathfrak{b}}\right\rangle \backslash \Sigma_{\mathfrak{a} \mathfrak{b}}^{+}(a, d)$, and multiplying $\sigma_{b}=C_{\mathfrak{b}}^{-1}\left(\begin{array}{ll}a & b \\ 0 & d\end{array}\right) C_{\mathfrak{a}} \in \Sigma_{\mathfrak{a b}}^{+}(a, d)$ on the left by $\gamma_{\mathfrak{b}}^{n}$ and on the right by $\gamma_{\mathfrak{a}}^{m}$ changes $b \mapsto b+m a+n d$. Therefore a set of representatives for $\left\langle\gamma_{\mathfrak{b}}\right\rangle\left\langle\Sigma_{\mathfrak{a} \mathfrak{b}}^{+}(a, d) /\left\langle\gamma_{\mathfrak{a}}\right\rangle\right.$ is

$$
\left\{\sigma_{b} \in \Sigma_{\mathfrak{a b}}^{+}(a, d): 0 \leqslant b<(a, d)\right\},
$$

while a set of representatives for $\left\langle\gamma_{\mathfrak{b}}\right\rangle \backslash \Sigma_{\mathfrak{a b}}^{+}(a, d)$, respectively $\Sigma_{\mathfrak{a} \mathfrak{b}}^{+}(a, d) /\left\langle\gamma_{\mathfrak{a}}\right\rangle$, is the same set, with the range for $b$ replaced by $0 \leqslant b<d$, respectively $0 \leqslant b<a$, proving (3.11). 
Using (3.11), formula (3.10) becomes

$$
\sum_{\sigma \in \Gamma \backslash \Sigma} \frac{a_{\mathfrak{a}}(\sigma)}{d_{\mathfrak{a}}(\sigma)}=\sum_{a, d>0} a \sum_{\mathfrak{b} \in C(\Gamma)} \frac{1}{a} \cdot\left|\Sigma_{\mathfrak{a} \mathfrak{b}}(a, d) / \Gamma_{\mathfrak{a}}\right|=|\Sigma / \Gamma|
$$

by the second decomposition in (3.8), and the claim follows from the equality $|\Sigma / \Gamma|=|\Gamma \backslash \Sigma|$.

We now introduce the cuspidal sum entering the trace formula on the Eisenstein subspace. Denote by $\Sigma_{\mathfrak{a}}(a, d)$ the set $\Sigma_{\mathfrak{a} a}(a, d)$ introduced in (3.10) and let $\Sigma_{\mathfrak{a}}=\Sigma_{\mathfrak{a} \mathfrak{a}}$ be the stabilizer of the cusp $\mathfrak{a}$ in $\Sigma$. We define the arithmetic function $\Phi_{\Gamma, \Sigma}^{\chi}(a, d)$ as in $(2.9)$ the introduction.

Theorem 3.5. Let $\Gamma$ be a Fuchsian group of the first kind, $k \geqslant 2$, and $\chi$ a character of $\Gamma$ with $\chi(-1)=(-1)^{k}$ if $-1 \in \Gamma$. Let $\Sigma \subset \widetilde{\Gamma}$ be a double coset satisfying Assumption 3.3.

(a) With the function $\Phi_{\Gamma, \Sigma}^{\chi}(a, d)$ defined above, we have

$$
\operatorname{Tr}\left([\Sigma], E_{k}(\Gamma, \chi)\right)=\sum_{a, d>0} a^{k-1} \Phi_{\Gamma, \Sigma}^{\chi}(a, d)-\delta_{k, 2} \delta_{\chi, \mathbf{1}} \sum_{\sigma \in \Gamma \backslash \Sigma} \tilde{\chi}(\sigma) .
$$

(b) The function $\Phi_{\Gamma, \Sigma}^{\chi}(a, d)$ is symmetric in $a, d$ and for $a \neq d$ we have

$$
\Phi_{\Gamma, \Sigma}^{\chi}(a, d)=\frac{1}{|d-a|} \sum_{\sigma \in H_{\Gamma, \Sigma}(a, d)} \operatorname{sgn}(\sigma)^{k} \widetilde{\chi}(\sigma)
$$

where $H_{\Gamma, \Sigma}(a, d) \subset \Sigma$ is a system of representatives for the hyperbolic $\Gamma$-conjugacy classes $X \subset \bar{\Sigma}$ whose elements fix two cusps of $\Gamma$, and that have eigenvalues $a, d$ or $-a,-d$.

Proof. (a) By (3.7), the action of $[\Sigma]$ on Eisenstein series corresponds to an action on $P \in \mathbb{C}^{|C(\Gamma, \chi)|}$ given by

$$
P \mid[\Sigma](\mathfrak{a})=\sum_{\sigma \in \Gamma \backslash \Sigma} P(\sigma \mathfrak{a}) \frac{a_{\mathfrak{a}}(\sigma)^{k-1}}{d_{\mathfrak{a}}(\sigma)} \tilde{\chi}(\sigma), \quad \mathfrak{a} \in \mathbb{C}^{|C(\Gamma, \chi)|}
$$

and we conclude

$$
\operatorname{Tr}\left([\Sigma], \mathbb{C}^{|C(\Gamma, \chi)|}\right)=\sum_{\mathfrak{a} \in C(\Gamma, \chi)} \sum_{\sigma \in \Gamma_{\mathfrak{a}} \backslash \Sigma_{\mathfrak{a}}} \frac{a_{\mathfrak{a}}(\sigma)^{k-1}}{d_{\mathfrak{a}}(\sigma)} \widetilde{\chi}(\sigma) .
$$

If $k=2$ and $\chi=\mathbf{1}$, let $P_{0} \in \mathbb{C}^{|C(\Gamma)|}$ such that $P_{0}(\mathfrak{a})=1$ for all $\mathfrak{a} \in C(\Gamma)$. We have that

$$
P_{0} \mid[\Sigma]=P_{0} \cdot \sum_{\sigma \in \Gamma \backslash \Sigma} \tilde{\chi}(\sigma)
$$

indeed, we can assume without loss of generality that $\Sigma=\Gamma \sigma_{0} \Gamma$ is a primitive double coset, so $\tilde{\chi}$ is constant on $\Sigma$ and by (3.14) we obtain that $P_{0} \mid[\Sigma](\mathfrak{a})$ is given by the left hand side of the identity in Lemma 3.4, multiplied by $\widetilde{\chi}\left(\sigma_{0}\right)$. The exact sequence (3.6) then gives

$$
\operatorname{Tr}\left([\Sigma], E_{k}(\Gamma, \chi)\right)=\operatorname{Tr}\left([\Sigma], \mathbb{C}^{|C(\Gamma, \chi)|}\right)-\delta_{k, 2} \delta_{\chi, \mathbf{1}} \sum_{\sigma \in \Gamma \backslash \Sigma} \widetilde{\chi}(\sigma) .
$$


We rewrite $(3.15)$ as $\operatorname{Tr}\left([\Sigma], \mathbb{C}^{|C(\Gamma, \chi)|}\right)=\sum_{a, d>0} a^{k-1} \Psi_{\Gamma, \Sigma}^{\chi}(a, d)$, with

$$
\Psi_{\Gamma, \Sigma}^{\chi}(a, d)=\frac{1}{d} \sum_{\mathfrak{a} \in C(\Gamma, \chi)} \sum_{\sigma \in \Gamma_{\mathfrak{a}} \backslash \Sigma_{\mathfrak{a}}(a, d)} \operatorname{sgn}(\sigma)^{k} \widetilde{\chi}(\sigma) .
$$

Since $\operatorname{sgn}(\sigma)^{k} \widetilde{\chi}(\sigma)$ is invariant under $\sigma \mapsto \gamma \sigma$ and $\sigma \mapsto \sigma \gamma$, for $\gamma \in \Gamma_{\mathfrak{a}}$ and $\mathfrak{a} \in C(\Gamma, \chi)$, and $\Sigma_{\mathfrak{a}}(a, d)$ is also left and right invariant under multiplication by $\Gamma_{\mathfrak{a}}$, by the first equality in (3.11) it follows that $\Psi_{\Gamma, \Sigma}^{\chi}=\Phi_{\Gamma, \Sigma}^{\chi}$, proving (3.12).

(b) That $\Phi_{\Gamma, \Sigma}^{\chi}$ is symmetric follows from (3.13), since the right hand side is obviously symmetric in $a, d$. To prove (3.13), denote by $\Theta_{\Gamma, \Sigma}^{\chi}(a, d)$ its right hand side. Each $\sigma \in H_{\Gamma, \Sigma}(a, d)$ fixes two cusps of $\Gamma$, and exactly one of them, denoted $\mathfrak{a}$, satisfies $C_{\mathfrak{a}} \sigma C_{\mathfrak{a}}^{-1}=\operatorname{sgn}(\sigma)\left(\begin{array}{ll}a & b \\ 0 & d\end{array}\right)$, independent of the scaling matrix $C_{\mathfrak{a}}$ used (for the other cusp, $a, d$ are reversed-see [15, p. 266]). By replacing $\sigma$ by a conjugate, we can assume that $\mathfrak{a}$ belongs to the set of cusp representatives $C(\Gamma)$ fixed in $\S 3.1 .1$, and $\pm \sigma \in \Sigma_{\mathfrak{a}}(a, d)$, with the minus sign possible if and only if $-1 \in \Gamma$ by Assumption 3.3. Denoting by $\Sigma_{\mathfrak{a}}^{\prime}(a, d)$ either $\Sigma_{\mathfrak{a}}^{+}(a, d)$ (defined in $\left.(3.9)\right)$ if $-1 \in \Gamma$, or $\Sigma_{\mathfrak{a}}(a, d)$ if $-1 \notin \Gamma$, we have by Lemma 3.6 that $\Sigma_{\mathfrak{a}}^{\prime}(a, d)$ consists of hyperbolic elements fixing two cusps of $\Gamma$, and we obtain

$$
\Theta_{\Gamma, \Sigma}^{\chi}(a, d)=\frac{1}{|d-a|} \sum_{\mathfrak{a} \in C(\Gamma)} \sum_{\sigma \in \Gamma_{\mathfrak{a}} \Downarrow \Sigma_{\mathfrak{a}}^{\prime}(a, d)} \operatorname{sgn}(\sigma)^{k} \tilde{\chi}(\sigma),
$$

where $\|$ denotes the conjugation action of $\Gamma_{\mathfrak{a}}$ on $\Sigma_{\mathfrak{a}}^{\prime}(a, d)$, and the sum is over any system of representatives for the orbits of this action. The set $\Sigma_{\mathfrak{a}}^{\prime}(a, d)$ is invariant under left and right multiplication by the group $\left\langle\gamma_{\mathfrak{a}}\right\rangle$ generated by $\gamma_{\mathfrak{a}}$. Changing variables $\sigma \mapsto \gamma \sigma$ for $\gamma \in\left\langle\gamma_{\mathfrak{a}}\right\rangle$ in the sum over $\sigma$, scales the sum by $\operatorname{sgn}(\gamma)^{k} \chi(\gamma)$. Therefore the inner sum vanishes, unless $\chi(\gamma)=\operatorname{sgn}(\gamma)^{k}$ for $\gamma \in \Gamma_{\mathfrak{a}}$, that is unless $\mathfrak{a} \in C(\Gamma, \chi)$. To show that $\Theta_{\Gamma, \Sigma}^{\chi}=\Phi_{\Gamma, \Sigma}^{\chi}$, it remains to prove that

$$
\frac{1}{|d-a|} \cdot\left|\Gamma_{\mathfrak{a}} \| \Sigma_{\mathfrak{a}}^{\prime}(a, d)\right|=\frac{1}{(a, d)} \cdot\left|\Gamma_{\mathfrak{a}} \backslash \Sigma_{\mathfrak{a}}(a, d) / \Gamma_{\mathfrak{a}}\right|,
$$

which follows by a similar argument as (3.11).

3.2. A trace formula on the cuspidal subspace. In order to extract from (2.5) the Eisenstein contribution, we will use an equivalent version, proved in [7], which can be stated for an arbitrary Fuchsian group of the first kind $\Gamma$.

For a $\bar{\Gamma}$-conjugacy class $X \subset \mathrm{GL}_{2}^{+}(\mathbb{R}) /\{ \pm 1\}$, we define the following analogue of the conjugacy class invariant $\varepsilon$ for $\Gamma$ :

$$
\varepsilon_{\Gamma}(X)=\left\{\begin{array}{cl}
\frac{|\Gamma \backslash \mathcal{H}|}{2 \pi} & \text { if } M_{X} \text { scalar } \\
\frac{\operatorname{sgn} \Delta(X)}{\left|\operatorname{Stab}_{\bar{\Gamma}} M_{X}\right|} & \text { otherwise }
\end{array}\right.
$$

where $|\Gamma \backslash \mathcal{H}|$ is the area of a fundamental domain for $\Gamma$ with respect to the standard hyperbolic metric, and we use the convention that $1 / \infty=0$. Any double coset $\Sigma \subset \widetilde{\Gamma}$ contains only finitely many conjugacy classes $X$ with $\varepsilon_{\Gamma}(X) \neq 0$, namely the elliptic, scalar, and those hyperbolic classes that contain an element fixing two distinct cusps of $\Gamma$, for which $\varepsilon_{\Gamma}(X)=1$ (see Lemma 3.6). We 
show in $[7$, Sec. 4$]$ that the trace formula in Theorem 1 is equivalent to the following statement:

$$
\begin{array}{r}
\operatorname{Tr}\left([\Sigma], M_{k}(\Gamma, \chi)+S_{k}^{c}(\Gamma, \chi)\right)=\sum_{X \subset \bar{\Sigma}} p_{k-2}\left(\operatorname{Tr} M_{X}, \operatorname{det} M_{X}\right) \tilde{\chi}\left(M_{X}\right) \varepsilon_{\Gamma}(X) \\
+\delta_{k, 2} \delta_{\chi, 1} \sum_{\sigma \in \Gamma \backslash \Sigma} \widetilde{\chi}(\sigma),
\end{array}
$$

where the sum is over $\bar{\Gamma}$-conjugacy classes $X$ in $\bar{\Sigma}$ with representative $M_{X} \in \Sigma$.

We now use Theorem 3.5 to obtain a trace formula on the cuspidal subspace from (3.17), after first recalling a result of J. Oesterlé.

Lemma 3.6 (Oesterlé). Let $\Gamma$ be a Fuchsian subgroup of the first kind and let $M \in \widetilde{\Gamma}$ such that $\mathrm{Stab}_{\bar{\Gamma}} M$ is finite. Then $M$ is either elliptic, or it is hyperbolic fixing two distinct cusps of $\Gamma$. In the latter case we have $\left|\operatorname{Stab}_{\bar{\Gamma}} M\right|=1$

Proof. More precisely, it is shown in [6, Proof of Theorem 2] that that any non-scalar $M \in \widetilde{\Gamma}$ with $\operatorname{Tr}^{2}(M) \geqslant 4 \operatorname{det}(M)$ falls in one of three cases: $M$ is parabolic fixing a cusp of $\Gamma ; M$ is hyperbolic with the same fixed points as those of a hyperbolic matrix in $\Gamma$; or $M$ is hyperbolic fixing two cusps. It immediately follows that $\operatorname{Stab}_{\Gamma} M$ is infinite in the first two cases, and $\left|\operatorname{Stab}_{\bar{\Gamma}} M\right|=1$ in the last case.

Note that formula (3.17) makes sense for an arbitrary Fuchsian group of the first kind with cusps and an arbitrary double coset $\Sigma$, and we indeed expect it to hold in this level of generality. We therefore state the next theorem so that formula (3.18) below holds whenever (3.17) does, under a mild assumption on the double coset $\Sigma$.

Theorem 3.7. Let $\Gamma$ be a Fuchsian subgroup of the first kind with cusps, and let $\Sigma \subset \widetilde{\Gamma}$ be a double coset satisfying Assumption 3.3. Then the trace formula (3.17) is equivalent to

$$
\begin{array}{r}
\operatorname{Tr}\left([\Sigma], S_{k}(\Gamma, \chi)+S_{k}^{c}(\Gamma, \chi)\right)=\sum_{X, \Delta(X) \leqslant 0} p_{k-2}\left(\operatorname{Tr} M_{X}, \operatorname{det} M_{X}\right) \widetilde{\chi}\left(M_{X}\right) \varepsilon_{\Gamma}(X) \\
-\sum_{a, d>0} \min (a, d)^{k-1} \Phi_{\Gamma, \Sigma}^{\chi}(a, d)+2 \delta_{k, 2} \delta_{\chi, \mathbf{1}} \sum_{\sigma \in \Gamma \backslash \Sigma} \tilde{\chi}(\sigma),
\end{array}
$$

where the sum is over $\Gamma$-conjugacy classes $X$ contained in $\bar{\Sigma}$, and $\Phi_{\Gamma, \Sigma}^{\chi}$ is defined in (2.8).

In particular, Theorem 2 holds under the assumptions of Theorem 1.

Just like the trace formula (3.17) is equivalent to that in Theorem 1, formula (3.18) is equivalent to that in Theorem 2 (see [7, Sec. 4] for the details). Note that by Remark 3.2, we can scale $\Sigma$ so that the sum over $a, d$ is over integers $a, d>0$ with $a d=\operatorname{det} M$, for some $M \in \Sigma$.

Proof. Let $\operatorname{Tr}_{>0}(\Gamma, \chi, \Sigma, k)$ be the sum in (3.17) over the conjugacy classes $X \subset \bar{\Sigma}$ with $\Delta(X)>$ 0 . Only the hyperbolic classes $X$ with representatives $M_{X} \in \Sigma$ fixing two (distinct) cusps of $\Gamma$ contribute to the sum, and $\varepsilon_{\Gamma}(X)=1$ for these classes, by Lemma 3.6. Let $H_{\Gamma, \Sigma}(a, d) \subset \Sigma$ be a system of representatives for these conjugacy classes that have eigenvalues $a, d$ or $-a,-d$. Since 
$p_{k-2}(a+d, a d)=\frac{d^{k-1}-a^{k-1}}{d-a}$, we obtain $($ recall $\operatorname{sgn}(\sigma)$ is the sign of the eigenvalues of $\sigma)$ :

$$
\begin{aligned}
\operatorname{Tr}_{>0}(\Gamma, \chi, \Sigma, k) & =\sum_{d>a>0} \frac{d^{k-1}-a^{k-1}}{d-a} \sum_{\sigma \in H_{\Gamma, \Sigma}(a, d)} \operatorname{sgn}(\sigma)^{k} \widetilde{\chi}(\sigma) \\
& =\sum_{d>a>0}\left(d^{k-1}-a^{k-1}\right) \Phi_{\Gamma, \Sigma}^{\chi}(a, d) \\
& =\operatorname{Tr}\left([\Sigma], E_{k}(\Gamma, \chi)\right)-\sum_{a, d>0} \min (a, d)^{k-1} \Phi_{\Gamma, \Sigma}^{\chi}(a, d)+\delta_{k, 2} \delta_{\chi, 1} \sum_{\sigma \in \Gamma \backslash \Sigma} \tilde{\chi}(\sigma),
\end{aligned}
$$

where the second equality follows from part (b), and the third from part (a) of Theorem 3.5, using also the symmetry of $\Phi_{\Gamma, \Sigma}^{\chi}$. The equivalence of the trace formulas (3.17) and (2.7) is now clear.

Remark 3.8. The sum over conjugacy classes $X$ with $\Delta(X)=0$ in (2.7) contains scalar classes only, by the definition of $\varepsilon_{\Gamma}(X)$, so it equals

$$
\frac{|\Gamma \backslash \mathcal{H}|}{2 \pi} \sum_{\lambda}(k-1) \lambda^{k-2} \widetilde{\chi}(\lambda I)
$$

where the sum is over $\lambda$ with $\lambda I \in \Sigma$ and $\lambda>0$ if $-1 \in \Gamma$.

3.3. Another formula for the cuspidal sum $\Phi_{\Gamma, \Sigma}^{\chi}$. Assume now that $\Gamma$ is a finite index subgroup of $\Gamma_{1}=\mathrm{SL}_{2}(\mathbb{Z})$. To compute explicitly the function $\Phi_{\Gamma, \Sigma}^{\chi}$ appearing in Theorems 3.5 and 2 , it is convenient to parametrize the cusps of $\Gamma$ by the space of double cosets $\Gamma \backslash \Gamma_{1} / \Gamma_{1 \infty}$, where $\Gamma_{1 \infty}$ denotes the stabilizer of the cusp $\infty$ in $\Gamma_{1}$.

Let $\chi$ be a character of $\Gamma, k \geqslant 2$, and assume that $\chi(-1)=(-1)^{k}$ if $-1 \in \Gamma$. Let $R(\Gamma) \subset \Gamma_{1}$ be a system of representatives for the cusp space $\Gamma \backslash \Gamma_{1} / \Gamma_{1 \infty}$, which is in bijection with a set of representatives $C(\Gamma)$ for $\Gamma$-equivalence classes of cusps of $\Gamma$ by $C \mapsto \mathfrak{a}=C \infty$. The set $C(\Gamma, \chi)$ introduced in $\S 3.1 .1$ is then in bijection with a set

$$
R(\Gamma, \chi)=\left\{C \in R(\Gamma) \mid \chi\left(\varepsilon C T^{j} C^{-1}\right)=\varepsilon^{k} \text { if } \varepsilon C T^{j} C^{-1} \in \Gamma \text { for some } \varepsilon \in\{ \pm 1\}\right\} .
$$

For $C \in R(\Gamma, \chi)$, let $\omega(C)$ be the smallest positive integer such that $C T^{\omega(C)} C^{-1} \in \pm \Gamma{ }^{5}$

Lemma 3.9. Let $\Sigma \subset \mathcal{M}$ be a double coset satifying Assumption 3.1. The function $\Phi_{\Gamma, \Sigma}^{\chi}$ introduced in (2.8) is given by

$$
\Phi_{\Gamma, \Sigma}^{\chi}(a, d)=\frac{1}{(a, d)} \sum_{C \in R(\Gamma, \chi)} \sum_{\substack{M \in\left\langle T^{\omega(C)}\right\rangle \backslash M_{a, d}^{\infty} /\left\langle T^{\omega(C)}\right\rangle \\ \pm C M C^{-1} \in \Sigma}}( \pm 1)^{k} \widetilde{\chi}\left( \pm C M C^{-1}\right),
$$

where $M_{a, d}^{\infty}=\left\{\left(\begin{array}{ll}a & b \\ 0 & d\end{array}\right) \in \mathcal{M}\right\}$.

Proof. For $C \in R(\Gamma, \chi)$, the stabilizer $\Gamma_{\mathfrak{a}}$ of the cusp $\mathfrak{a}=C \infty$ is generated by $\gamma_{\mathfrak{a}}= \pm C T^{\omega(C)} C^{-1} \in$ $\Gamma$, and as scaling matrix we can take

$$
C_{\mathfrak{a}}=\left(\begin{array}{cc}
\omega(C)^{-1 / 2} & 0 \\
0 & \omega(C)^{1 / 2}
\end{array}\right) C^{-1} .
$$

\footnotetext{
${ }^{5}$ That is, $\omega(C)$ is the width of the cusp $C$ if $C T^{\omega(C)} C^{-1} \in \Gamma$, or it is half the width if $-1 \notin \Gamma$ and $C T^{\omega(C)} C^{-1} \in-\Gamma$.
} 
The bijection $R(\Gamma, \chi) \simeq C(\Gamma, \chi)$ given by $C \mapsto \mathfrak{a}=C \infty$, then yields a bijection

$$
\left\{M \in\left\langle T^{\omega(C)}\right\rangle \backslash M_{a, d}^{\infty} /\left\langle T^{\omega(C)}\right\rangle: \pm C M C^{-1} \in \Sigma\right\} \longrightarrow \Gamma_{\mathfrak{a}} \backslash \Sigma_{\mathfrak{a}}(a, d) / \Gamma_{\mathfrak{a}},
$$

given by $M \mapsto \pm C M C^{-1} \in \Sigma$, where the sign can be chosen positive if $-1 \in \Gamma$, and only one choice is possible if $-1 \notin \Gamma$ (since $\Sigma \cap(-\Sigma)=\emptyset$ by Assumption 3.1). We conclude that the right hand sides of (3.20) and (2.8) are equal term by term.

\section{Explicit trace formulas for $\Gamma_{0}(N)$}

We now specialize $\Gamma=\Gamma_{0}(N)$ and we compute the functions $\mathcal{C}_{\Gamma, \Sigma}^{\chi}, \Phi_{\Gamma, \Sigma}^{\chi}$ in $(2.6),(2.8)$ to prove Theorems 3 and 4 in the introduction. We use the formula for $\Phi_{\Gamma, \Sigma}^{\chi}$ given in (3.20). In Section 4.1 we consider the usual Hecke operators for $S_{k}(\Gamma, \chi)$, while in Section 4.2 we consider a composition of Hecke and Atkin-Lehner operators on $S_{k}(\Gamma)$. In Section 4.3 we prove Corollary 1.

4.1. Trace of Hecke operators on $\Gamma_{0}(N)$ with Nebentypus. We take $\Gamma=\Gamma_{0}(N), \Sigma=\Delta_{n}$ as in (2.3), and $\chi, \tilde{\chi}$ defined there. If $\chi(-1)=(-1)^{k}$, we have $\mathcal{C}_{\Gamma, \Sigma}^{\chi}(M)=\mathcal{C}_{N, \chi}(M)$, defined in (2.17). The function $\mathcal{C}_{N, \chi}(M)$ was computed explicitly by Oesterlé [6, Eq.(35)], and it satisfies Assumption 2.1. We sketch the proof in the next lemma, since we will use it later. Recall that for $u\left|N, u^{2}\right| t^{2}-4 n$, in the introduction we have defined the set

$$
S_{N}(u, t, n)=\left\{\alpha \in(\mathbb{Z} / N \mathbb{Z})^{\times}: \alpha^{2}-t \alpha+n \equiv 0 \quad(\bmod N u)\right\} .
$$

Lemma 4.1 (Oesterlé). For $M=\left(\begin{array}{cc}A & B \\ C\end{array}\right) \in \mathcal{M}_{n}$, let $t=\operatorname{Tr}(M)$, and $u=(G, N)$ where $G$ is the content of the quadratic form $Q_{M}=[C, D-A,-B]$. Then

$$
\mathcal{C}_{N, \chi}(M)=B_{N, \chi}(u, t, n):=\frac{\varphi_{1}(N)}{\varphi_{1}(N / u)} \sum_{\alpha \in S_{N}(u, t, n)} \chi(\alpha) .
$$

Proof. For $X=\left(\begin{array}{ll}a & b \\ c & d\end{array}\right) \in \Gamma_{1}$, we have $X M X^{-1}=\left(\begin{array}{cc}\alpha & -Q_{M}(-b, a) \\ Q_{M}(-d, c) & t-\alpha\end{array}\right)$, where $\alpha$ satisfies

$$
\alpha^{2}-t \alpha+n=Q_{M}(-d, c) Q_{M}(-b, a) .
$$

The condition $X M X^{-1} \in \Delta_{n}$ is equivalent to $Q_{M}(d,-c) \equiv 0(\bmod N)$ and $(\alpha, N)=1$, so we have $\alpha \in S_{N}(u, t, n)$, where we set $u=(G, N)$. Moreover, $\alpha$ satisfies $M\left(\begin{array}{c}-d \\ c\end{array}\right) \equiv \alpha\left(\begin{array}{c}-d \\ c\end{array}\right)(\bmod N)$, that is

$$
\alpha d \equiv d A-c B(\bmod N), \quad \alpha c \equiv c D-d C(\bmod N) .
$$

which determines its class mod $N$ uniquely depending only on the point $(c: d) \in \mathbb{P}^{1}(\mathbb{Z} / N \mathbb{Z})$, namely on the coset of $X$ in $\Gamma_{0}(N) \backslash \Gamma_{1}$. Set $\mathcal{S}_{N}(M)=\left\{X \in \Gamma_{0}(N) \backslash \Gamma_{1}: X M X^{-1} \in \Delta_{n}\right\}$. We have therefore a well-defined map

$$
\tau: \mathcal{S}_{N}(M) \longrightarrow S_{N}(u, t, n), \quad X \mapsto a_{X M X^{-1}},
$$

and one can show that $\left|\tau^{-1}(\alpha)\right|=\varphi_{1}(N) / \varphi_{1}(N / u)$ independent of $\alpha \in S_{N}(u, t, n)$, finishing the proof. We refer to $[6]$ for the details.

To compute the function $\Phi_{N, \chi}:=\Phi_{\Gamma_{0}(N), \Delta_{n}}^{\chi}$ in Theorem 2, we use Lemma 3.9. 
Lemma 4.2. Let $k \geqslant 2, N \geqslant 1$, and $\chi$ a character of conductor $c_{\chi} \mid N$ such that $\chi(-1)=(-1)^{k}$. We have

$$
\Phi_{N, \chi}(a, d)=\sum_{\substack{N=r s \\(r, s) \mid(N / c(\chi), a-d)}} \varphi((r, s)) \chi\left(\alpha_{r, s}^{a, d}\right)
$$

where $\alpha=\alpha_{r, s}^{a, d}$ is the unique solution $\bmod \frac{r s}{(r, s)}$ of $\alpha \equiv a(\bmod r), \alpha \equiv d(\bmod s)$.

Proof. Since $-1 \in \Gamma$, formula (3.20) gives

$$
\Phi_{N, \chi}(a, d)=\frac{1}{(a, d)} \sum_{C \in R(\Gamma, \chi)} \sum_{\substack{M \in\left\langle T^{\omega(C)}\right\rangle \backslash M_{a, d}^{\infty} /\left\langle T^{\omega(C)}\right\rangle \\ C M C^{-1} \in \Delta}} \chi\left(a_{C M C^{-1}}\right) .
$$

We choose the set of representatives $R(\Gamma)$ for $\Gamma \backslash \Gamma_{1} / \Gamma_{1 \infty}$ to consist of matrices $C=\left(\begin{array}{c}p * \\ r\end{array}\right)$ with $N=r s$, and $q$ running through a set $\mathcal{S}_{r}$ with $\left|\mathcal{S}_{r}\right|=\varphi((r, s))$. The condition $\chi\left(C T^{j} C^{-1}\right)=1$ whenever $C T^{j} C^{-1} \in \Gamma$ in (3.19) is the same as $\chi(1+p r j)=1$ if $N \mid r^{2} j$, namely if $\frac{N}{(r, s)} \mid r j$. This can happen if and only if $\chi$ has conductor $c_{\chi} \mid \frac{N}{(r, s)}$, hence $R(\Gamma, \chi)$ consists of those $C \in R(\Gamma)$ as above with $(r, s) \mid\left(N / c_{\chi}\right)$. The width of the cusp $C \infty$ is $\omega(C)=s /(r, s)$.

Let $\alpha=a_{C M C^{-1}}$ for a fixed $C \in R(\Gamma, \chi)$ as above, and set $M=\left(\begin{array}{ll}a & b \\ 0 & d\end{array}\right)$ with $b$ running through the residues modulo $\omega(C) g$, where $g=(a, d)$. By (4.2), the condition $C M C^{-1} \in \Delta$ is equivalent to:

$$
\alpha r \equiv d r \quad(\bmod N), \quad \alpha q \equiv a q-b r \quad(\bmod N), \quad(\alpha, N)=1 .
$$

Therefore $\alpha \equiv d(\bmod s), \alpha \equiv a(\bmod r)$, which determines $\alpha$ uniquely modulo $r s /(r, s)$, thus it determines $\chi(\alpha)$ uniquely since $c_{\chi} \mid \frac{N}{(r, s)}$. We also have $(r, s) \mid(a-d)$ and since $(s, g)=1$, there are $g$ solutions $b(\bmod g s /(r, s))$ of the congruence $b \equiv q(a-\alpha) / r(\bmod s)$, independent of $q \in \mathcal{S}_{r}$. We conclude

$$
\Phi_{N, \chi}(a, d)=\sum_{N=r s} \chi(\alpha) \cdot\left|\mathcal{S}_{r}\right|
$$

with $\alpha=\alpha_{r, s}^{a, d},\left|\mathcal{S}_{r}\right|=\varphi((r, s))$, and $(r, s)|(a-d),(r, s)| N / c_{\chi}$ in the summation range.

4.2. Trace of Atkin-Lehner and Hecke operators for $\Gamma_{0}(N)$. Let $\Gamma=\Gamma_{0}(N)$ and $\chi=1$ the trivial character. For $N=\ell \ell^{\prime}$ with $\left(\ell, \ell^{\prime}\right)=1$ consider the double coset $\Theta_{\ell}=\Gamma w_{\ell} \Gamma$, where $w_{\ell}=\left(\begin{array}{cc}\ell x & y \\ N z & \ell t\end{array}\right) \in \mathcal{M}_{\ell}$ with $x, y, z, t \in \mathbb{Z}$. The coset space $\Gamma \backslash \Theta_{\ell}$ consists of one element $\Gamma w_{\ell}$, and the Atkin-Lehner involution $W_{\ell}$ on $M_{k}(\Gamma)$ is given by $W_{\ell}=\ell^{-w / 2}\left[\Theta_{\ell}\right]$ with $w=k-2$. We let $n \geqslant 1$ be arbitrary and consider the composition of Hecke and Atkin-Lehner operators $T_{n} \circ W_{\ell}=\frac{1}{\ell^{w / 2}}\left[\Delta_{n} \Theta_{\ell}\right]$. We have

$$
\Delta_{n} \Theta_{\ell}=\left\{\left(\begin{array}{ll}
a & b \\
c & d
\end{array}\right) \in \mathcal{M}_{\ell n}: N|c, \ell| \operatorname{Tr}(M), \ell \mid a,\left(a, \ell^{\prime}\right)=1,(b, \ell)=1\right\} .
$$

The double coset $\Theta_{\ell} \Delta_{n}$ is characterized by the same conditions, except that $(b, \ell)=1$ is replaced by $(c / N, \ell)=1$. If $(n, \ell)=1$, the last two conditions are empty, so the double cosets $\Delta_{n}, \Theta_{\ell}$ commute, but that is not the case when $(n, \ell)>1$, when the double cosets, and the corresponding operators, do not commute. If $(n, \ell)>1$ the double coset $\Theta_{\ell} \Delta_{n}$ does not satisfy Assumption 3.1.

Lemma 4.3. The double coset $\Delta_{n} \Theta_{\ell}$ satisfies Assumption 3.1. 
Proof. Let $\Sigma=\Delta_{n} \Theta_{\ell}$. We have to show that if $\gamma=\left(\begin{array}{cc}x & y \\ z & t\end{array}\right) \in \Gamma_{1}$ and $\sigma=\left(\begin{array}{ll}a & b \\ c & d\end{array}\right) \in \Sigma$ such that $\gamma \sigma \in \Sigma$, then we have $\gamma \in \Gamma_{0}(N)$. Indeed, we have $\ell \ell^{\prime} \mid a z$ and $\ell \mid b z$ and since $\left(a, \ell^{\prime}\right)=1,(b, \ell)=1$, we conclude that $\ell \ell^{\prime} \mid z$, so $\gamma \in \Gamma_{0}(N)$.

For $\Gamma=\Gamma_{0}(N)$ and $\Sigma=\Delta_{n} \Theta_{\ell}$, assuming $k \geqslant 2$ is even we write

$$
\mathcal{C}_{\Gamma, \Sigma}^{1}(M)=\mathcal{C}_{N, \ell}(M):=\#\left\{A \in \Gamma_{0}(N) \backslash \Gamma_{1}: A M A^{-1} \in \Delta_{n} \Theta_{\ell}\right\} .
$$

In the next lemma we compute $\mathcal{C}_{N, \ell}$ and see that it satisfies Assumption 2.1.

Lemma 4.4. For $M \in \mathcal{M}_{\ell n}$, set $t=\operatorname{Tr}(M)$, and let $G$ be the content of the associated quadratic form $Q_{M}$. Then the coefficient $\mathcal{C}_{N, \ell}(M)$ vanishes if $\ell \nmid t$ and for $\ell \mid t$ it is given by

$$
\mathcal{C}_{N, \ell}(M)=\delta_{(\ell, G), 1} \cdot \mathcal{C}_{\ell^{\prime}, \mathbf{1}}(M)=\sum_{\substack{u\left|(\ell, G) \\ u^{\prime}\right|\left(\ell^{\prime}, G\right)}} C_{\ell^{\prime}}\left(u^{\prime}, t, \ell n\right) \mu(u),
$$

where $\mathcal{C}_{\ell^{\prime}, \mathbf{1}}(M)$ is defined in in Lemma 4.1 and $C_{\ell^{\prime}}(u, t, n):=C_{\ell^{\prime}, \mathbf{1}}(u, t, n)$, for $\mathbf{1}$ the trivial character modulo $\ell^{\prime}$.

The coefficients $C_{\ell^{\prime}}(u, t, n)$ are computed explicitly in Lemma 4.5.

Proof. Let $g M g^{-1}=\left(\begin{array}{ll}\alpha & \beta \\ \gamma & \delta\end{array}\right)$. By (4.4) and (4.5) we have

$$
\mathcal{C}_{N, \ell}(M)=\left|\left\{g \in \Gamma \backslash \Gamma_{1}: \ell|\operatorname{Tr}(M), \ell| \alpha, \ell \ell^{\prime} \mid \gamma,\left(\alpha, \ell^{\prime}\right)=1,(\beta, \ell)=1\right\}\right| .
$$

Assuming from now on $\ell \mid \operatorname{Tr}(M)$ (otherwise the previous set is empty), let $M=\left(\begin{array}{l}A \\ C\end{array} \underset{D}{B}\right)$, and $g=\left(\begin{array}{ll}a & b \\ c & d\end{array}\right) \in \Gamma_{1}$. The coset of $g$ in $\Gamma \backslash \Gamma_{1}$ is identified with the point $(c: d) \in \mathbb{P}^{1}\left(\mathbb{Z} / \ell \ell^{\prime} \mathbb{Z}\right)$. By (4.2), the conditions $\ell|\gamma, \ell| \alpha$ are equivalent to

$$
d A \equiv c B \quad(\bmod \ell), \quad d C \equiv c D \quad(\bmod \ell) .
$$

Since $\beta=-Q_{M}(-b, a)$, the condition $(\beta, \ell)=1$ implies $(G, \ell)=1$. Conversely, if $p \mid(\beta, \ell)$ it follows that $p$ divides all entries of $g M g^{-1}$, so $p \mid G$. Therefore the condition $(\beta, \ell)=1$ is equivalent to $(G, \ell)=1$.

Since $\left(\ell, \ell^{\prime}\right)=1$, the Chinese remainder theorem gives

$$
\mathcal{C}_{N, \ell}(M)=\mathcal{C}_{\ell^{\prime}, \mathbf{1}}(M) \cdot N_{\ell}(M)
$$

with $\mathcal{C}_{\ell^{\prime}, \mathbf{1}}(M)$ given by (2.18) for the trivial character $1 \bmod \ell^{\prime}$, and $N_{\ell}(M)$ equals 0 if $(G, \ell)>1$, while $N_{\ell}(M)$ denotes the number of solutions $(c: d) \in \mathbb{P}^{1}(\mathbb{Z} / \ell \mathbb{Z})$ of the system $(4.6)$ if $(G, \ell)=1$. As $A D-B C=n \ell$, we have $(G, \ell)=1$ if and only if $(A, B, C, D, \ell)=1$, and in this case $N_{\ell}(M)=1$. This proves the first equality, and the second follows by writing $\delta_{(\ell, G), 1}=\sum_{u \mid(\ell, G)} \mu(u)$.

Lemma 4.5. For any $N \geqslant 1$ and for $u\left|N, u^{2}\right| t^{2}-4 n$ we have

$$
C_{N}(u, t, n)=\left|S_{N}(t, n)\right| \cdot C_{N}\left(u, t^{2}-4 n\right)
$$

where $S_{N}(t, n)=\left\{\alpha \in(\mathbb{Z} / N \mathbb{Z})^{\times}: \alpha^{2}-t \alpha+n \equiv 0(\bmod N)\right\}$, and the coefficients $C_{N}(u, D)$, defined for $u\left|N, u^{2}\right| D$, are multiplicative in $(N, u)$, namely

$$
C_{N}(u, D)=\prod_{p \mid N} C_{p^{\nu p(N)}}\left(p^{\nu_{p}(u)}, D\right) .
$$


If $N=p^{a}$ with $p$ prime and $a \geqslant 1$ we have $C_{N}\left(p^{0}, D\right)=1, C_{N}\left(p^{a}, D\right)=p^{\left\lceil\frac{a}{2}\right\rceil}$, and setting $b=\nu_{p}(D)$ (with $b=\infty$ if $D=0$ ), for $0<i<a$ we have: if $p$ is odd then

$$
C_{N}\left(p^{i}, D\right)= \begin{cases}p^{\left\lceil\frac{i}{2}\right\rceil}-p^{\left\lceil\frac{i}{2}\right\rceil-1} & \text { if } 1 \leqslant i \leqslant b-a, i \equiv a(\bmod 2) \\ -p^{\left\lceil\frac{i}{2}\right\rceil-1} & \text { if } i=b-a+1, i \equiv a(\bmod 2) \\ p^{\left\lfloor\frac{i}{2}\right\rfloor}\left(\frac{D / p^{b}}{p}\right) & \text { if } i=b-a+1, i \neq \equiv(\bmod 2) \\ 0 & \text { otherwise, }\end{cases}
$$

while if $p=2$ then

$$
C_{N}\left(2^{i}, D\right)= \begin{cases}2^{\left\lceil\frac{i}{2}\right\rceil-1} & \text { if } 1 \leqslant i \leqslant b-a-2, i \equiv a(\bmod 2) \\ -2^{\left\lceil\frac{i}{2}\right\rceil-1} & \text { if } i=b-a-1, i \equiv a(\bmod 2) \\ 2^{\left\lceil\frac{i}{2}\right\rceil-1} \epsilon_{4}\left(D / 2^{b}\right) & \text { if } i=b-a, i \equiv a(\bmod 2) \\ 2^{\left\lfloor\frac{i}{2}\right\rfloor}\left(\frac{D / 2^{b}}{2}\right) & \text { if } i=b-a+1, i \equiv \equiv(\bmod 2) \text { and } D / 2^{b} \equiv 1(\bmod 4) \\ 0 & \text { otherwise, }\end{cases}
$$

where $(\dot{\bar{p}})$ denotes the Legendre symbol, and $\epsilon_{4}$ is the notrivial quadratic character mod 4.

Proof. If $S_{N}(t, n)=\emptyset$, then $S_{N}(u, t, n)=\emptyset$ for all $u \mid N$, so the constants $C_{N}(u, t, n)$ are all 0 . Since $\left|S_{N}(u, t, n)\right|=\left|S_{N}(u,-t, n)\right|$, we can write

$$
C_{N}(u, t, n)=\left|S_{N}(t, n)\right| \cdot C_{N}(u, D)
$$

for some function $C_{N}(u, D)$. The function $C_{N}(u, D)$ is multiplicative in $N$, as $C_{N, \mathbf{1}}(u, t, n)$ and $\left|S_{N}(t, n)\right|$ are multiplicative, and, by (2.18), if $N=p^{a}$ it equals

$$
C_{N}\left(p^{i}, D\right)=\frac{\varphi_{1}\left(p^{a}\right) / \varphi_{1}\left(p^{a-i}\right) \cdot\left|S_{N}\left(p^{i}, t, n\right)\right|-\varphi_{1}\left(p^{a}\right) / \varphi_{1}\left(p^{a-i+1}\right) \cdot\left|S_{N}\left(p^{i-1}, t, n\right)\right|}{\left|S_{N}(t, n)\right|},
$$

with the second term in the numerator missing if $i=0$. The cardinality of the set $S_{p^{a}}\left(p^{i}, t, n\right)$ is straightforward to compute, leading to the formulas above.

To finish the proof of Theorem 4, we compute the function $\Phi_{N, \ell}:=\Phi_{\Gamma, \Delta_{n} \Theta_{\ell}}^{\chi=1}$ in Theorem 3.9.

Lemma 4.6. We have $\Phi_{N, \ell}(a, d)=0$ unless $n \ell=$ ad and $\ell \mid a+d$, when

$$
\Phi_{N, \ell}(a, d)=\frac{\varphi(\ell)}{\ell} \sum_{\substack{\ell^{\prime}=r s,(r, s) \mid a-d \\(r, a)=1,(s, d)=1}} \varphi((r, s)) .
$$

Proof. Letting $M_{b}=\left(\begin{array}{ll}a & b \\ 0 & d\end{array}\right)$ and $g=\operatorname{gcd}(a, d)$, by Lemma 3.9 we have

$$
\Phi_{N, \ell}(a, d)=\frac{1}{g} \sum_{C \in R(\Gamma)} \#\left\{b(\bmod g \omega(C)): C M_{b} C^{-1} \in \Delta_{n} \Theta_{\ell}\right\},
$$

where $R(\Gamma) \subset \Gamma_{1}$ is the set of representatives for the cusp space $\Gamma \backslash \Gamma_{1} / \Gamma_{1 \infty}$ chosen in the proof of Lemma 4.2. Counting elements in the sets above can now be done as in the proof of Lemma 4.2. We omit the proof, which can be found in our arXiv preprint $1408.4998 \mathrm{v} 2$. 
4.3. Proof of Corollary 1. The function $\Psi_{4}(a, d)$ is easily computable, so it remains to compute the sum over $t$ in Theorem 5 . We write it as:

$$
\sum_{0 \leqslant t \leqslant \sqrt{4 n}}^{\prime} p_{k-2}(t, n) \cdot \sum_{u \mid 4} H\left(\frac{4 n-t^{2}}{u^{2}}\right)\left(D_{4}(u, t, n)+(-1)^{k} D_{4}(u,-t, n)\right)
$$

with the prime indicating the the term with $t=0$ has coefficient $1 / 2$. The sum is restricted to those $t$ with $4 \mid \pm t-n-1$, as otherwise $D_{4}(u, \pm t, n)=0$. For $n$ even this means that $t$ is odd so only $u=1$ is present in the sum over $u$, obtaining the formula in part b). For $n$ odd, it follows that $t=2 s$ is even and we have a few cases depending on whether $\nu_{2}(2 s-n-1)$ is 2,3 or $\geqslant 4$. We leave the details to the reader, noting only that in the case $k$ even and $n$ odd we also use the class number relations in the following table, valid for $D \geqslant 0$ :

$$
\begin{array}{c|c|c}
D \equiv 3(\bmod 8) & D \equiv 7(\bmod 8) & D \equiv 0(\bmod 4) \\
H(4 D)=4 H(D) & H(4 D)=2 H(D) & H(4 D)=3 H(D)-2 H(D / 4)
\end{array}
$$

\section{REFERENCES}

[1] H. Cohen, Sums involving the values at negative integers of L-functions of quadratic characters, Math. Ann. 217 (1975), 271-285

[2] H. Cohen, Trace des opérateurs de Hecke sur $\Gamma_{0}(N)$. Séminaire de Théorie des Nombres de Bordeau, 1976-1977, 4-01-4-09

[3] M. Eichler, Über die Darstellbarkeit von Modulformen durch Thetareihen, J. Reine Angew. Math. 195 (1955), $156-171$

[4] H. Hijikata, Explicit formula of the traces of Hecke operators for $\Gamma_{0}(N)$, J. Math. Soc. Japan 26, No.1 (1974), $56-82$

[5] M.H. Mertens, Mock modular forms and class number relations. Research in the Math. Sciences 1:6 (2014)

[6] J. Oesterlé, Sur la trace des opérateurs de Hecke, Thèse de troisième cycle, Université Paris-Sud, Orsay (1977)

[7] A.A. Popa, On the trace formula for Hecke operators on congruence subgroups. Preprint, arXiv:1408.4998v4

[8] A.A. Popa, D. Zagier, A simple proof of the Eichler-Selberg trace formula. Draft available upon request

[9] A.A. Popa, D. Zagier, A combinatorial refinement of the Kronecker-Hurwitz class number relation. Proc. Amer. Math. Soc. 145/3 (2017), 1003-1008

[10] F. Rădulescu, Endomorphisms of spaces of virtual vectors fixed by a discrete group. Russian Math. Surveys 71 (2016), 291-343

[11] H. Saito, On Eichler's trace formula, J. Math. Soc. Japan 24 (1972), 333-340

[12] A. Selberg, Harmonic analysis and discontinuous groups in weakly symmetric Riemannian spaces with applications to Dirichlet series, J. Indian Math. Soc. (N.S.) 20 (1956), 47-87

[13] J.-P. Serre, Répartition asymptotique des valeurs propres de l'opérateur de Hecke $T_{p}$. Journal of the AMS 10 (1997), 75-102

[14] G. Shimura, Introduction to the arithmetic theory of automorphic functions, Princeton U. Press (1971)

[15] G. Shimura, On the trace formula for Hecke operators. Acta Math., vol. 132 (1974), 245-281

[16] N.-P. Skoruppa, D. Zagier, Jacobi forms and a certain space of modular forms. Inv. Math. 94 (1988), 113-146

[17] D. Zagier, Hecke operators and periods of modular forms. Israel math. conf. proc. Vol. 3 (1990), 321-336

[18] D. Zagier, Periods of modular forms, traces of Hecke operators, and multiple zeta values. Research into automorphic forms and $L$-functions (Kyoto, 1992). Sūrikaisekikenkyūsho Kōkyūroku 843 (1993), 162-170

Institute of Mathematics "Simion Stoilow" of the Romanian Academy, P.O. Box 1-764, RO-014700 Bucharest, ROMANIA

E-MAIL: AAPOPA@GMAIL.COM 